\title{
IMPULSIVE CONSENSUS FOR COMPLEX DYNAMICAL NETWORKS WITH NONIDENTICAL NODES AND COUPLING TIME-DELAYS*
}

\author{
BIN LIU ${ }^{\dagger}$ AND DAVID J. HILL ${ }^{\ddagger}$
}

\begin{abstract}
This paper investigates the problem of global consensus between a complex dynamical network $(\mathrm{CDN})$ and a known goal signal by designing an impulsive consensus control scheme. The dynamical network is complex with respect to the uncertainties, nonidentical nodes, and coupling time-delays. The goal signal can be a measurable vector function or a solution of a dynamical system. By utilizing the Lyapunov function and Lyapunov-Krasovskii functional methods, robust global exponential stability criteria are derived for the error system, under which global exponential impulsive consensus is achieved for the CDN. These criteria are expressed in terms of linear matrix inequalities (LMIs) and algebraic inequalities. Thus, the impulsive controller can be easily designed by solving the derived inequalities. Meanwhile, the estimations of the consensus rate for global exponential consensus are also obtained. Two examples with numerical simulations are worked out for illustration.
\end{abstract}

Key words. complex dynamical networks, synchronization, impulsive consensus, global exponential impulsive consensus, robust global exponential stability, consensus rate, time-delays, Lyapunov-Krasovskii function

AMS subject classifications. 93C30, 34K34, 34K45, 93D15, 93C65, 93C95

DOI. $10.1137 / 080722060$

1. Introduction. Synchronization of chaotic systems and its potential applications to secure communication has been an active research area since the 1990s. Numerous methods have been developed for chaos synchronization (see, for example, $[1,2,3,4,5,6,7,8,9,10,11,49])$. Recently, the more general problem of synchronization of complex dynamical networks $(\mathrm{CDNs})$ has been studied in the literature (see, for example, $[12,13,14,15,16,17,18,19,20,21,22,23,24,25,26,27])$. A CDN consists of coupled nodes, which are usually dynamical systems. Several approaches have been proposed for synchronization of a CDN; for example, feedback control synchronization (see, for example, [22, 23]), adaptive synchronization [20], synchronization based on the invariance principle [10], the state-observer-based approach [26], and the impulsive synchronization scheme [31, 35, 54], etc.

It has been noticed that the synchronization of CDNs studied in the literature has limitations such as the following: (i) the coupled nodes have the same dynamics; (ii) by using the linearization technique and matrix eigenvalue method, most synchronization criteria are local; and (iii) uncertainty and time-delays which are common in practical CDNs have not been studied fully, although there are published results $[8,15,20,35]$ which study the robust synchronization problem with respect to uncertainties, and some results $[21,23,24,53]$ deal with a single constant time-delay.

*Received by the editors April 23, 2008; accepted for publication (in revised form) December 1, 2010; published electronically March 4, 2011. This work was supported by the ARC-Australia (DP0881391). A preliminary version of this paper appears in Proceedings of the 47th IEEE Conference on Decision and Control, Cancun, Mexico, December 9-11, 2008, pp. 2258-2263.

http://www.siam.org/journals/sicon/49-2/72206.html

${ }^{\dagger}$ Research School of Information Sciences and Engineering, The Australian National University, Canberra, ACT 0200, Australia (bin.liu@anu.edu.au). This author was supported in part by Hunan University of Technology, Hunan, China, under the Foundation of NSFC-China (60874025).

${ }^{\ddagger}$ School of Electrical and Information Engineering, The University of Sydney, Sydney, NSW 2006, Australia (davidh@ee.usyd.edu.au). 
Uncertainties often occur due to parameter errors, modeling mismatches, measurement errors, approximations, channel noises, etc. And time-delays commonly occur due to the congestion of the network traffic and the fact that the switching and spreading speed of the hardware and circuit implementation is finite. Moreover, the time-delays presented in many real synchronization schemes are difficult to know a priori, are in the form of multiple time-delays, and are time-varying.

The CDNs with nonidentical nodes represent more general and practical networks than the models typically studied in the literature. For example, in an ecological species network, even if all the nodes belong to the same species, different individuals may have different characteristics and dynamic behaviors, and thus this species network cannot be modeled as a network with identical nodes. Moreover, some abrupt events (impulses) such as flu pandemic, war, etc., will lead to changes of species population and dynamic behavior in the network. For another example, here we consider military systems. Suppose one wants to use a missile network to attack the enemy's aircraft carrier in the ocean. Obviously, it needs many missiles from different military bases, and the target is composed of the static carrier and the aircraft (which might not be static) on it. We can look at all the missiles as nodes in the network, and the nodes from different bases will have different flying trajectories. Hence, this missile network is a CDN with nonidentical nodes. Moreover, it is necessary to adjust (via impulsive adjustment control) the trajectories of all missiles (nodes) before the network hits the target (achieves the consensus). So it is important to study the dynamical properties for CDNs with nonidentical nodes. However, to the best of our knowledge, no literature has been published for the consensus control problem of CDNs with nonidentical nodes. Obviously, allowing different dynamics of nodes in a CDN brings difficulties in achieving consensus. Without control, it has been shown recently how to quantify the ultimate bounds on the synchronization errors as related to the diversity of the node dynamics [42]. If uncertainty and time-delays occur simultaneously in a CDN with nonidentical nodes, and consensus is to be achieved to a known goal function, it will be much more difficult to use previous synchronization control schemes, especially for the global consensus problem. Hence, there is a need to study new consensus control schemes which can achieve the objective.

In this paper, we propose an impulsive consensus control scheme for the consensus problem between CDNs and the known signal. In this control scheme, the control signal is designed to be input into the $\mathrm{CDN}$ as follows: at impulsive instances, the impulse signal is input into the nodes, and at other times, the signal containing the goal signal is input into the nodes. Hence, this control scheme is a type of impulsive hybrid control scheme. Impulsive control arises naturally from a wide variety of applications, such as drug administration, spacecraft control, inspection processes in operations research, and native forest ecosystems management, just to name a few. Based on the stability theory of impulsive systems (see [28, 29, 30, 34, 36, 37, 38, 39, 40] and references therein), the impulsive control method (see [31, 32, 33, 34, 35, 46, 47, 48] and references therein) provides a greater prospect for solving many problems that are basically defined by continuous dynamical systems, but on which only discretetime actions are exercised. An essential benefit of the impulsive control approach may be derived from the fact that such controls are typically simpler to implement and cheaper. In $[9,11]$, impulsive control was introduced to synchronize chaotic systems. Recently, impulsive synchronization for CDNs was also reported in [35]. Theory and experiments have proved that the impulsive synchronization scheme for chaotic systems or CDNs (with identical nodes) has good robustness against uncertainties and can achieve global synchronization. 
The aim of this paper is to study the global exponential impulsive consensus (GEIC) problem for CDNs and a known goal function. The model of CDNs consists of the nonidentical nodes, uncertainties, and coupling time-delays. Here, the "uncertainties" means the uncertain parameters, which take values in some intervals. The exponential consensus scheme has an obvious advantage over other consensus schemes in that the consensus speed and consensus time can be estimated easily. By utilizing the Lyapunov function and Lyapunov-Krasovskii functional [41, 42] methods, robust global exponential stability results for delay error systems shall be established, and then we shall derive several criteria under which the GEIC is achieved for the uncertain CDN with nonidentical nodes and coupling time-delays. These criteria are expressed in terms of LMIs (linear matrix inequalities) and algebraic inequalities. Thus, the conditions of consensus are easy to test. Moreover, the solutions of the LMIs and algebraic inequalities give rise directly to impulsive controllers to achieve GEIC.

The organization of this paper is as follows. In sections 2 and 3, we define some notation and give some preliminaries. In section 4 , impulsive consensus criteria are established. The impulsive consensus control is designed in section 5. Two representative examples are given in section 6 . Section 7 concludes the paper.

2. Notation. We list the notation and symbols used in this paper as follows:

Let $\mathbb{R}^{n}$ denote the $n$-dimensional Euclidean space, and let $\mathbb{R}_{+}=[0,+\infty), \mathbb{Z}=$ $\{0,1,2, \ldots\}$, and let $\|\cdot\|$ be the Euclidean norm. Let $I$ be the identity matrix. Matrix $X>(\geq,<, \leq) 0$ means that $X$ is a symmetric positive definite (positive semidefinite, negative definite, negative semidefinite) matrix. Denote by $\lambda_{\max }(\cdot)\left(\lambda_{\min }(\cdot)\right)$ the maximal (minimal) eigenvalue of matrix (.). For a positive real number $\tau>0$ and any $\varphi \in C\left(\left[t_{0}-\tau, t_{0}\right], \mathbb{R}^{n}\right]$, we define $\|\varphi\|_{\tau} \triangleq \sup _{t_{0}-\tau \leq s \leq t_{0}}\{\|\varphi(s)\|\}$.

For a sequence $\left\{t_{k}, k \in \mathbb{Z}\right\}$ satisfying $0 \leq t_{0}<t_{1}<\cdots<t_{k}<t_{k+1}<\cdots$, let $\Delta_{k} \triangleq t_{k+1}-t_{k}, k \in \mathbb{Z}, \Delta_{\text {sup }} \triangleq \sup _{k \in \mathbb{Z}}\left\{\Delta_{k}\right\}, \Delta_{\text {inf }} \triangleq \inf _{k \in \mathbb{Z}}\left\{\Delta_{k}\right\}, S\left[t_{0}, t\right] \triangleq \max \{k:$ $\left.t_{0} \leq t_{k} \leq t\right\}$ for any $t \geq t_{0}$.

Denote $\Sigma^{*} \triangleq\left\{\Sigma \in \mathbb{R}^{n^{2} \times n^{2}}: \Sigma=\operatorname{diag}\left(\epsilon_{11}, \ldots, \epsilon_{n^{2} n^{2}}\right),\left|\epsilon_{i j}\right| \leq 1 ; i, j=1,2, \ldots, n\right\}$, and define $N[U, V]=\left\{\left(s_{i j}\right) \in \mathbb{R}^{n \times n}: u_{i j} \leq s_{i j} \leq v_{i j}, i, i=1,2, \ldots, n\right\}$ as the interval matrix for known matrices $U=\left(u_{i j}\right)_{n \times n}$ and $V=\left(v_{i j}\right)_{n \times n}$.

The following abbreviations are used in the paper:

GAS: globally asymptotically stable.

RGAS: robustly globally asymptotically stable.

GES: globally exponentially stable.

RGES: robustly globally exponentially stable.

GEIC: global exponential impulsive consensus.

GIC: global impulsive consensus.

3. Preliminaries. Consider the uncertain CDN with $N$ nonidentical nodes and coupling time-delays,

$$
S_{i}: \quad \dot{x}_{i}=f_{i}\left(t, x_{i}\right)+g_{i}\left(x_{1}\left(t-h_{i}\right), \ldots, x_{N}\left(t-h_{i}\right)\right), \quad i=1,2, \ldots, N,
$$

where $x=\left(x_{1}^{T}, x_{2}^{T}, \ldots, x_{n}^{T}\right)^{T} \in \mathbb{R}^{n N} ; x_{i}=\left(x_{i 1}, x_{i 2}, \ldots, x_{i n}\right)^{T} \in \mathbb{R}^{n}$ represents the state of the $i$ th node $S_{i} ; f_{i}: \mathbb{R}_{+} \times \mathbb{R}^{n} \rightarrow \mathbb{R}^{n}$ is a smooth nonlinear vector function; $g_{i}: \mathbb{R}^{n N} \rightarrow \mathbb{R}^{n}$ is a smooth nonlinear vector function representing the coupling of the $i$ th node with other nodes; and $h_{i}$ represents the coupling time-delay of the signal transmitted from the network to the $i$ th node, where $h_{i}$ satisfies $0 \leq h_{i} \leq \tau$ for some constant $\tau>0$ and $i=1,2, \ldots, N$. We call the system (3.1) an uncertain CDN based 
on the fact that there are uncertainties in functions $f_{i}, g_{i}, i=1,2, \ldots, N$. In this paper, we use the following assumption.

Assumption 3.1. Assume that $f_{i}\left(t, x_{i}\right)=A_{i} x_{i}+\tilde{f}_{i}\left(t, x_{i}\right)$, where $A_{i} \in \mathbb{R}^{n \times n}$ is an interval matrix with $A_{i} \in N\left[A_{i_{1}}, A_{i_{2}}\right]$ for known matrices $A_{i_{1}}$ and $A_{i_{2}}$, and functions $\tilde{f}_{i}, i=1, \ldots, N$, satisfy

$$
\left\|\tilde{f}_{i}\left(t, s_{1}\right)-\tilde{f}_{i}\left(t, s_{2}\right)\right\| \leq L_{i}\left\|s_{1}-s_{2}\right\|, \quad i=1, \ldots, N,
$$

for some positive constants $L_{i}>0, i=1, \ldots, N$, and for all $t \in \mathbb{R}_{+}$.

It should be noted that when the network (3.1) achieves consensus, namely, when the states $x_{i}(t) \rightarrow s(t), i=1,2, \ldots, N$, as $t \rightarrow \infty$, the coupling terms should vanish, i.e., $g_{i}(s, s, \ldots, s)=0, i=1,2, \ldots, N$. Thus, we give the following assumption on function $g_{i}$.

Assumption 3.2. Assume that $g_{i}\left(x_{1}, \ldots, x_{N}\right)=\sum_{j=1}^{N} B_{i j} x_{j}+\tilde{g}_{i}\left(x_{1}, \ldots, x_{N}\right)$, where $B_{i j} \in \mathbb{R}^{n \times n}$ with $B_{i j} \in N\left[B_{i j_{1}}, B_{i j_{2}}\right]$ for known matrices $B_{i j_{1}}$ and $B_{i j_{2}}$, and functions $\tilde{g}_{i}$ satisfy $\tilde{g}_{i}(s, s, \ldots, s)=0, i=1, \ldots, N$, respectively, for some nonnegative constants $M_{i j} \geq 0, i, j=1, \ldots, N$,

$$
\begin{aligned}
& \sum_{j=1}^{N} B_{i j}=0, \quad i=1,2, \ldots, N \\
& \left\|\tilde{g}_{i}\left(x_{1}, \ldots, x_{N}\right)-\tilde{g}_{i}\left(y_{1}, \ldots, y_{N}\right)\right\| \leq \sum_{j=1}^{N} M_{i j}\left\|x_{j}-y_{j}\right\| .
\end{aligned}
$$

Problem formulation. Let $s(t)$ be a given measurable smooth vector function satisfying $s(t) \in \mathbb{R}^{n}$ for any $t \in \mathbb{R}_{+}$. The aim of this paper is to design an impulsive hybrid control scheme for CDN (3.1) such that consensus among the node states $x_{i}(t)$ $(i=1,2, \ldots, N)$ and $s(t)$ can be achieved.

Consider the uncertain CDN (3.1) under impulsive consensus control,

$$
\dot{x}_{i}=f_{i}\left(t, x_{i}\right)+g_{i}\left(x_{1}\left(t-h_{i}\right), \ldots, x_{N}\left(t-h_{i}\right)\right)+u_{i}\left(t, x_{i}, s\right), \quad i=1, \ldots, N,
$$

where $\left\{u_{i}\left(t, x_{i}, s\right), i=1,2, \ldots, N\right\}$ is the impulsive hybrid controller designed as (see Figure 3.1)

$$
u_{i}\left(t, x_{i}, s\right)=\dot{s}-f_{i}(t, s)+\sum_{k=1}^{\infty} \delta\left(t-t_{k}\right)\left(C_{i_{k}}-I\right)\left(x_{i}(t)-s(t)\right),
$$

where $C_{i_{k}} \in \mathbb{R}^{n \times n}, k \in \mathbb{Z}$, are impulsive control gain matrices to be designed; $\left\{t_{k}, k \in\right.$ $\mathbb{Z}\}$ are the impulsive instances satisfying $0 \leq t_{0}<t_{1}<t_{2}<\cdots$, with $\Delta_{\text {sup }}<\infty$ and $\lim _{k \rightarrow \infty} t_{k}=\infty$; and $\delta(\cdot)$ is the Dirac delta function.

Figure 3.1 depicts the entire impulsive control scheme for consensus ("impulsive consensus scheme" for short) between the known signal $s(t)$ and CDN (3.1) with coupling time-delays, where $S_{i}$ stands for the $i$ th node, $S$ generates the objective vector function $s(t)$, and $g_{i}$ is the delay network coupling of the $i$ th node, $i=1,2, \ldots, N$. In this control scheme, the control signal is input into the CDN as follows: at impulsive instance $t_{k}$, the impulse signal $\left(C_{i_{k}}-I\right)\left(x_{i}\left(t_{k}\right)-s\left(t_{k}\right)\right)$ is input into the node $S_{i}$, and at other nonimpulse times $t \neq t_{k}$, the signal $\dot{s}-f_{i}(t, s)$ containing the goal signal is input into the node $S_{i}$ of the CDN.

Copyright (c) by SIAM. Unauthorized reproduction of this article is prohibited. 


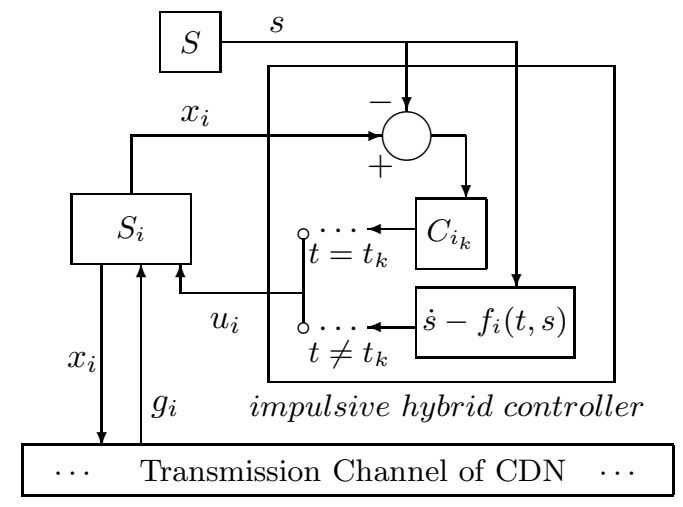

FIG. 3.1. Impulsive consensus scheme of $S_{i}$.

By the property of the Dirac delta function $\delta(\cdot)$ and assuming that $x\left(t^{+}\right)=x(t)$, (3.4) is equivalent to the following system:

$$
\left\{\begin{array}{l}
\dot{x}_{i}=f_{i}\left(t, x_{i}\right)+g_{i}\left(x_{1}\left(t-h_{i}\right), \ldots, x_{N}\left(t-h_{i}\right)\right)+\dot{s}-f_{i}(t, s), t \in\left[t_{k}, t_{k+1}\right), \\
\Delta x_{i}\left(t_{k}\right)=\left(C_{i_{k}}-I\right)\left(x_{i}\left(t_{k}\right)-s\left(t_{k}\right)\right), t=t_{k}, k \in \mathbb{Z}, i=1,2, \ldots, N
\end{array}\right.
$$

where $\Delta x_{i}\left(t_{k}\right)=x\left(t_{k}\right)-x\left(t_{k}^{-}\right), k \in \mathbb{Z}, i=1,2, \ldots, N$.

Define the consensus errors as $e_{i}(t):=x_{i}(t)-s(t)$. Then, by Assumptions 3.1 and 3.2 , one has an error dynamical system of the form

$$
\left\{\begin{array}{l}
\dot{e}_{i}=A_{i} e_{i}+\hat{f}_{i}\left(t, x_{i}, s\right)+\sum_{j=1}^{N} B_{i j} e_{j}\left(t-h_{i}\right)+\hat{g}_{i}\left(x\left(t-h_{i}\right), s\right), \\
\left.\Delta e_{i}=\left(C_{i_{k}}-I\right) e_{i}(t), \quad t=t_{k}, k \in \mathbb{Z}, i=1,2, \ldots, N, \quad t \in t_{k}, t_{k+1}\right),
\end{array}\right.
$$

where $\hat{f}_{i}\left(t, x_{i}, s\right)=\tilde{f}_{i}\left(t, x_{i}\right)-\tilde{f}_{i}(t, s)$ and $\hat{g}_{i}\left(x\left(t-h_{i}\right), s\right)=\tilde{g}_{i}\left(x_{1}\left(t-h_{i}\right), \ldots, x_{N}(t-\right.$ $\left.\left.h_{i}\right)\right)-\tilde{g}_{i}\left(s\left(t-h_{i}\right), \ldots, s\left(t-h_{i}\right)\right), i=1,2, \ldots, N$.

Remark 3.1. It should be noted that system (3.1) represents a more general CDN than that considered in the literature in the following sense:

1. The nodes in (3.1) are nonidentical with uncertainties; i.e., functions $f_{i}(i=$ $1,2, \ldots, N)$ can be different and uncertain.

2. If function $s(t)$ satisfies $\dot{s}=f_{i}(t, s), i=1,2, \ldots, N$, i.e., $s(t)$ is the common solution of every single node, then the impulsive consensus scheme is to make all states of the nonidentical nodes approach the same solution $s(t)$.

3. If all $f_{i}$ are the same, i.e., $f_{i}=f, i=1,2, \ldots, N$, and $s(t)$ is a solution of system $\dot{y}=f(t, y)$, then the consensus problem is the synchronization problem discussed in the literature; see, for example, [20, 21, 22, 23, 24, 25, 26, 27].

4. In the above mentioned literature on CDNs, the coupling coefficient matrices have the special form $B_{i j}=c C_{i j} \Gamma$, where the constant $c>0$ denotes the coupling strength, $\Gamma=\operatorname{diag}\left\{r_{1}, r_{2}, \ldots, r_{n}\right\}$, and $C=\left(C_{i j}\right)_{N \times N}$ are the coupling matrices of network nodes. And only a single time-delay $\tau$, i.e., $h_{i}=\tau, i=1, \ldots, N$, is considered.

Remark 3.2. In the above impulsive consensus scheme, since the target $s$ is known, there are no couplings in $u_{i}$ between node $S_{i}$ and other nodes, $i=1,2, \ldots, N$. If one wants to design the control scheme with couplings in it (that is, a distributed control scheme), then we can revise $u_{i}$ as $\hat{u}_{i}$ :

$\hat{u}_{i}(t, x, s)=\dot{s}-f_{i}(t, s)+\sum_{k=1}^{\infty} \delta\left(t-t_{k}\right)\left[\left(C_{i i_{k}}-I\right)\left(x_{i}(t)-s(t)\right)+\sum_{j=1, j \neq i}^{N} C_{i j_{k}}\left(x_{j}(t)-s(t)\right)\right]$. 
Clearly, if the consensus target $s$ is not known a priori, but equations $\dot{s}=f_{i}(t, s)$, $i=1,2, \ldots, N$, have a common solution $s$, the control $\hat{u}_{i}$ with couplings is changed to $\tilde{u}_{i}: \tilde{u}_{i}=\sum_{k=1}^{\infty} \sum_{j=1}^{N} \delta\left(t-t_{k}\right) C_{i j_{k}}\left(x_{j}(t)-s(t)\right)$. For these two distributed control schemes with couplings in them, the consensus issue between $x_{i}, i=1,2, \ldots, N$, and $s$ can be studied similarly as the case for $u_{i}$ used in this paper.

It should be noted that if $s$ is not specified and $\dot{s}=f_{i}(t, s), i=1,2, \ldots, N$, do not have any common solution, then, for the consensus issue of CDN (3.1), the distributed control scheme is necessary. In this case, letting $\bar{x}(t)=\frac{1}{N} \sum_{i=1}^{N} x_{i}(t)$, we can design the impulsive distributed control $\bar{u}_{i}$ as

$$
\bar{u}_{i}(t, x, \bar{x})=\sum_{k=1}^{\infty} \delta\left(t-t_{k}\right)\left[\left(C_{i i_{k}}-I\right)\left(x_{i}(t)-\bar{x}(t)\right)+\sum_{j=1, j \neq i}^{N} C_{i j_{k}}\left(x_{j}(t)-\bar{x}(t)\right)\right] .
$$

Then the aim is to achieve consensus between $x_{i}, i=1,2, \ldots, N$, and $\bar{x}$. This case is beyond the scope of this paper and is the subject of current work on impulsive consensus.

By [36], for any $X \in N\left[X_{1}, X_{2}\right], X$ can be formulated as

$$
X=X_{0}+\Delta X=X_{0}+E \Sigma F,
$$

where $X_{0}=\frac{1}{2}\left(X_{1}+X_{2}\right), H=\frac{1}{2}\left(X_{2}-X_{1}\right)=\left(h_{i j}\right)_{n \times n}, E E^{T}=\operatorname{diag}\left\{\sum_{j=1}^{n} h_{1 j}, \ldots\right.$, $\left.\sum_{j=1}^{n} h_{n j}\right\}, F^{T} F=\operatorname{diag}\left\{\sum_{j=1}^{n} h_{j 1}, \ldots, \sum_{j=1}^{n} h_{j n}\right\}, \Sigma \in \Sigma^{*}$.

Assumption 3.3. For interval matrices $A_{i}, B_{i j}$ in CDN (3.1), there exist known matrices $E, F_{A_{i}}, F_{i j}$ such that for any $\Sigma \in \Sigma^{*}, i, j=1,2, \ldots, N$,

$$
\left[\begin{array}{ll}
\Delta A_{i} & \Delta B_{i j}
\end{array}\right]=E \Sigma\left[\begin{array}{ll}
F_{A_{i}} & F_{i j}
\end{array}\right] .
$$

DEFINITION 3.1. The error system (3.6) is said to be RGAS if, for any initial condition, $\phi \in C\left[\left[t_{0}-\tau, t_{0}\right], \mathbb{R}^{n N}\right]$, any $A_{i} \in N\left[A_{i_{1}}, A_{i_{2}}\right], B_{i j} \in N\left[B_{i j_{1}}, B_{i j_{2}}\right]$, and for any time-delays $h_{j}$ with $0 \leq h_{j} \leq \tau$, the trivial solution of (3.6) is GAS.

DEFINITION 3.2. The error system (3.6) is said to be RGES with decay rate $\alpha$ if, for any initial condition, $\phi \in C\left[\left[t_{0}-\tau, t_{0}\right], \mathbb{R}^{n N}\right]$, any $A_{i} \in N\left[A_{i_{1}}, A_{i_{2}}\right], B_{i j} \in$ $N\left[B_{i j_{1}}, B_{i j_{2}}\right]$, and for any time-delays $h_{j}$ with $0 \leq h_{j} \leq \tau$, the trivial solution of (3.6) is GES; i.e., there exist two positive numbers $\alpha>0, K>0$ such that

$$
\|e(t)\| \leq K\|\phi\|_{\tau} e^{-\alpha\left(t-t_{0}\right)}, \quad t \geq t_{0},
$$

where $\phi(t)=\left(\phi_{1}^{T}(t), \ldots, \phi_{N}^{T}(t)\right)^{T} \in \mathbb{R}^{n N}, \phi_{i}(t) \in \mathbb{R}^{n}$, and $\|\phi\|_{\tau}^{2}=\sum_{i=1}^{N}\left\|\phi_{i}\right\|_{\tau}^{2}$.

DEFINITION 3.3. The impulsive consensus scheme is said to achieve GEIC at rate $\alpha$ if, for any initial condition $\phi$, the error system (3.6) is RGES with decay rate $\alpha$. If the system (3.6) is RGAS, then we say the network (3.1) can achieve GIC.

Remark 3.3. In the GEIC scheme, the consensus speed or consensus time can be estimated by using the decay rate, while in the GIC scheme this estimation is not presented.

Lemma 3.1 (see [43]). Let $E \in \mathbb{R}^{n \times n^{2}}, F \in \mathbb{R}^{n^{2} \times n}$, and $Y \in \mathbb{R}^{n \times n}$ be a symmetric matrix. Then, for any $\Sigma \in \Sigma^{*}$,

$$
Y+E \Sigma F+F^{T} \Sigma E^{T}<0
$$

holds if and only if there exists a positive constant $\epsilon>0$ such that

$$
Y+\epsilon E E^{T}+\epsilon^{-1} F^{T} F<0
$$

Copyright $@$ ㅇ by SIAM. Unauthorized reproduction of this article is prohibited. 
4. GEIC properties of CDNs. In this section, two types of GEIC properties of CDN (3.1) will be investigated. The first aims to show how impulses affect the consensus property, and the second how impulses can contribute to the achievement of consensus. $\Sigma \in \Sigma^{*}$.

By Assumption 3.3, we denote $A_{i}=A_{i_{0}}+E \Sigma F_{A_{i}}$ and $B_{i j}=B_{i j_{0}}+E \Sigma F_{i j}$, where

THEOREM 4.1. Suppose that Assumptions 3.1-3.3 hold and that there exist positive definite matrices $P_{i} \in \mathbb{R}^{n \times n}$ and constants $\epsilon_{i j}>0, \varepsilon_{i}>0, \alpha_{i}<0, i, j=$ $1,2, \ldots, N$, such that

(i) there exist some positive constants $\nu_{i}>0, \mu_{i}>0$, satisfying

$$
\nu_{i} I \leq P_{i} \leq \mu_{i} I, \quad i=1,2, \ldots, N
$$

(ii) for $i=1,2, \ldots, N$, the following LMIs hold:

$$
\left(\begin{array}{cccccc}
\Psi_{i}\left(A_{i_{0}}\right)-\alpha_{i} P_{i} & P_{i} B_{i 1_{0}} & \cdots & P_{i} B_{i N_{0}} & P_{i} E & F_{A_{i}}^{T} \\
B_{i 1_{0}}^{T} P_{i} & -\epsilon_{i 1} I & \cdots & 0 & 0 & F_{i 1}^{T} \\
\vdots & \vdots & \ddots & \vdots & \vdots & \vdots \\
B_{i N_{0}}^{T} P_{i} & 0 & \cdots & -\epsilon_{i N} I & 0 & F_{i N}^{T} \\
E^{T} P_{i} & 0 & \cdots & 0 & -\varepsilon_{i}^{-1} I & 0 \\
F_{A_{i}} & F_{i 1} & \cdots & F_{i N} & 0 & -\varepsilon_{i} I
\end{array}\right)<0
$$

where $\Psi_{i}\left(A_{i_{0}}\right)=P_{i} A_{i_{0}}+A_{i 0}^{T} P_{i}+2 L_{i} \sqrt{\frac{\mu_{i}}{\nu_{i}}} P_{i}+\sum_{j=1}^{N} M_{i j} \epsilon_{i j}^{-1}\left\|P_{i}\right\| I$, and $\alpha_{i}, \epsilon_{i j}$ satisfy

$$
-\max _{1 \leq i \leq N}\left\{\alpha_{i}\right\}>\sum_{i=1}^{N} \max _{1 \leq j \leq N}\left\{\frac{\hat{\epsilon}_{i j}}{\nu_{j}}\right\}
$$

where $\hat{\epsilon}_{i j}=\epsilon_{i j}+M_{i j} \epsilon_{i j}\left\|P_{i}\right\|$;

(iii) there exist positive constants $\delta>1, \sigma>0$ satisfying

$$
\begin{aligned}
& \frac{\ln \beta_{k}}{\sigma}<\tau \delta \leq \Delta_{\text {inf }}, \quad k \in \mathbb{Z}, \\
& \sigma+a+b e^{\sigma \tau}=0
\end{aligned}
$$

where $\beta_{k}=\max _{1 \leq i \leq N}\left\{\lambda_{\max }\left(P_{i}^{-1} C_{i_{k}}^{T} P_{i} C_{i_{k}}\right)\right\}, a=\max _{1 \leq i \leq N}\left\{\alpha_{i}\right\}$, and $b=$ $\sum_{i=1}^{N} \max _{1 \leq j \leq N}\left\{\frac{\hat{\epsilon}_{i j}}{\nu_{j}}\right\}$. Then, the error system (3.6) is RGES with decay rate $\alpha \triangleq \frac{1}{2}\left\{\sigma-\frac{\ln M}{\delta \tau}\right\}$, and hence the $C D N$ (3.1) can achieve GEIC with $s(t)$ at rate $\alpha$, where $M=\sup _{k \in \mathbb{Z}}\left\{\beta_{k}, e^{\sigma \tau}\right\}$.

Proof. Let the Lyapunov function candidate be $V(e)=\sum_{i=1}^{N} e_{i}^{T} P_{i} e_{i}$. For $t \in$ $\left[t_{k}, t_{k+1}\right)$, taking the Dini derivative of $V(e(t))$ along the trajectory of system (3.6), we get

$$
D^{+} V(e(t))=\sum_{i=1}^{N}\left\{2 e_{i}^{T}(t) P_{i}\left(A_{i} e_{i}(t)+\hat{f}_{i}+\sum_{j=1}^{N} B_{i j} e_{j}\left(t-h_{i}\right)+\hat{g}_{i}\left(x\left(t-h_{i}\right), s\right)\right)\right\} .
$$

Copyright $@$ by SIAM. Unauthorized reproduction of this article is prohibited. 
By condition (i) and Assumptions 3.1-3.3, we get

$$
\begin{aligned}
& 2 e_{i}^{T} P_{i} \hat{f}_{i} \leq 2 \sqrt{e_{i}^{T} P_{i} e_{i}} \sqrt{e_{i}^{T} \hat{f}_{i}^{T} P_{i} \hat{f}_{i}} \leq 2 L_{i} \sqrt{\frac{\mu_{i}}{\nu_{i}}} e_{i}^{T} P_{i} e_{i}, \\
& 2 e_{i}^{T} P_{i} \hat{g}_{i}\left(x\left(t-h_{i}\right), s\right) \leq \sum_{j=1}^{N} M_{i j} \epsilon_{i j}^{-1}\left\|P_{i}\right\| e_{i}^{T} e_{i} \\
& \quad+\sum_{j=1}^{N} M_{i j} \epsilon_{i j}\left\|P_{i}\right\| e_{j}^{T}\left(t-h_{i}\right) e_{j}\left(t-h_{i}\right),
\end{aligned}
$$

where $\epsilon_{i j}>0(i, j=1,2, \ldots, N)$ are some positive constants.

It follows from (4.6)-(4.8) that, for $t \in\left[t_{k}, t_{k+1}\right)$,

$$
\begin{array}{r}
D^{+} V(e(t)) \leq \sum_{i=1}^{N}\left(\begin{array}{c}
e_{i}(t) \\
e_{1}\left(t-h_{i}\right) \\
\vdots \\
e_{N}\left(t-h_{i}\right)
\end{array}\right)^{T}\left(\begin{array}{cccc}
\Psi_{i}\left(A_{i}\right) & P_{i} B_{i 1} & \cdots & P_{i} B_{i N} \\
B_{i 1}^{T} P_{i} & -\epsilon_{i 1} I & \cdots & 0 \\
\vdots & \vdots & \ddots & \vdots \\
B_{i N}^{T} P_{i} & 0 & \cdots & -\epsilon_{i N} I
\end{array}\right)\left(\begin{array}{c}
e_{i}(t) \\
e_{1}\left(t-h_{i}\right) \\
\vdots \\
e_{N}\left(t-h_{i}\right)
\end{array}\right) \\
+\sum_{i=1}^{N} \sum_{j=1}^{N} \hat{\epsilon}_{i j} e_{j}^{T}\left(t-h_{i}\right) e_{j}\left(t-h_{i}\right)
\end{array}
$$

where $\Psi_{i}\left(A_{i}\right)=P_{i} A_{i}+A_{i}^{T} P_{i}+2 L_{i} \sqrt{\frac{\mu_{i}}{\nu_{i}}} P_{i}+\sum_{j=1}^{N} M_{i j} \epsilon_{i j}^{-1}\left\|P_{i}\right\| I$ and $\hat{\epsilon}_{i j}=\epsilon_{i j}+$ $M_{i j} \epsilon_{i j}\left\|P_{i}\right\|$.

By Assumption 3.3, for $i=1,2, \ldots, N$, we get

$$
\begin{aligned}
\Lambda_{i} \triangleq & \left(\begin{array}{cccc}
\Psi_{i}\left(A_{i}\right)-\alpha_{i} P_{i} & P_{i} B_{i 1} & \cdots & P_{i} B_{i N} \\
B_{i 1}^{T} P_{i} & -\epsilon_{i 1} I & \cdots & 0 \\
\vdots & \vdots & \ddots & \vdots \\
B_{i N}^{T} P_{i} & 0 & \cdots & -\epsilon_{i N} I
\end{array}\right) \\
& =\left(\begin{array}{cccc}
\Psi_{i}\left(A_{i_{0}}\right)-\alpha_{i} P_{i} & P_{i} B_{i 1_{0}} & \cdots & P_{i} B_{i N_{0}} \\
B_{i 1_{0}}^{T} P_{i} & -\epsilon_{i 1} I & \cdots & 0 \\
\vdots & \vdots & \ddots & \vdots \\
B_{i N_{0}}^{T} P_{i} & 0 & \cdots & -\epsilon_{i N} I
\end{array}\right)^{T} \\
& +\left(\begin{array}{c}
P_{i} E \\
0 \\
\vdots \\
0
\end{array}\right) \Sigma\left(\begin{array}{c}
F_{A_{i}}^{T} \\
F_{i 1}^{T} \\
\vdots \\
F_{i N}^{T}
\end{array}\right)^{T}+\left(\begin{array}{c}
F_{A_{i}}^{T} \\
F_{i 1}^{T} \\
\vdots \\
F_{i N}^{T}
\end{array}\right) \Sigma^{T}\left(\begin{array}{c}
P_{i} E \\
0 \\
\vdots \\
0
\end{array}\right)^{T}
\end{aligned}
$$

By Lemma 3.1, for every $i=1,2, \ldots, N$, we get that $\Lambda_{i}<0$ holds if and only if there exists positive constant $\varepsilon_{i}>0$ such that

$$
\Lambda_{i}+\varepsilon_{i}\left(\begin{array}{c}
P_{i} E \\
0 \\
\vdots \\
0
\end{array}\right)\left(\begin{array}{c}
P_{i} E \\
0 \\
\vdots \\
0
\end{array}\right)^{T}+\varepsilon_{i}^{-1}\left(\begin{array}{c}
F_{A_{i}}^{T} \\
F_{i 1}^{T} \\
\vdots \\
F_{i N}^{T}
\end{array}\right)\left(\begin{array}{c}
F_{A_{i}}^{T} \\
F_{i 1}^{T} \\
\vdots \\
F_{i N}^{T}
\end{array}\right)^{T}<0 .
$$

Copyright (c) by SIAM. Unauthorized reproduction of this article is prohibited. 
By the Schur complement theorem [45], for $i=1,2, \ldots, N$, the inequality (4.11) is equivalent to (4.2). Thus, for $t \in\left[t_{k}, t_{k+1}\right.$ ), by condition (ii), we get that

$$
\begin{aligned}
D^{+} V(e(t)) & \leq \sum_{i=1}^{N} \alpha_{i} e_{i}^{T} P_{i} e_{i}+\sum_{i=1}^{N} \sum_{j=1}^{N} \hat{\epsilon}_{i j} e_{j}^{T}\left(t-h_{i}\right) e_{j}\left(t-h_{i}\right) \\
& \leq \max _{1 \leq i \leq N}\left\{\alpha_{i}\right\} \cdot V(e(t))+\sum_{i=1}^{N} \max _{1 \leq j \leq N}\left\{\frac{\hat{\epsilon}_{i j}}{\nu_{j}}\right\} \cdot \bar{V}(t),
\end{aligned}
$$

where $\bar{V}(t)=\max _{-\tau \leq \theta \leq 0}\{V(e(t+\theta))\}$.

Hence, by the Halanay lemma and condition (iii), there exists a constant $\sigma>0$ such that

$$
V(e(t)) \leq \bar{V}\left(t_{k}\right) e^{-\sigma\left(t-t_{k}\right)}, \quad t \in\left[t_{k}, t_{k+1}\right)
$$

where $\bar{V}\left(t_{k}\right)=\sup _{t_{k}-\tau \leq s \leq t_{k}}\{V(s)\}$ and $\sigma>0$ satisfies (4.5).

When $t=t_{k}$, by condition (iii), we have

$$
V\left(e\left(t_{k}\right)\right)=\sum_{i=1}^{N} e_{i}^{T}\left(t_{k}^{-}\right) C_{i_{k}}^{T} P_{i} C_{i_{k}} e_{i}\left(t_{k}^{-}\right) \leq \beta_{k} V\left(x\left(t_{k}^{-}\right)\right),
$$

where $\beta_{k}=\max _{1 \leq i \leq N}\left\{\lambda_{\max }\left(P_{i}^{-1} C_{i_{k}}^{T} P_{i} C_{i_{k}}\right)\right\}, k \in \mathbb{Z}$.

Denote $\mu=\max _{1 \leq i \leq N}\left\{\mu_{i}\right\}$ and $M=\sup _{k \in \mathbb{Z}}\left\{\beta_{k}, e^{\sigma \tau}\right\}$. We show, by induction, that

$$
V(e(t)) \leq \mu M^{k-1}\|\phi\|_{\tau}^{2} e^{-\sigma\left(t-t_{0}\right)}, \quad t \in\left[t_{k-1}, t_{k}\right), k \in N .
$$

When $k=1$, since for all $t \in\left[t_{0}-\tau, t_{0}\right],\left\|e_{i}(t)\right\| \leq\left\|\phi_{i}\right\|_{\tau}=\sup _{t_{0}-\tau \leq t \leq t_{0}}\left\|\phi_{i}(t)\right\|$, we get for all $t \in\left[t_{0}-\tau, t_{0}\right], V(e(t)) \leq \max _{1 \leq i \leq N}\left\{\lambda_{\max }\left(P_{i}\right)\right\} \cdot \sum_{i=1}^{N}\left\|e_{i}(t)\right\|^{2} \leq \mu \cdot\|\phi\|_{\tau}^{2}$, where $\|\phi\|_{\tau}^{2}=\sum_{i=1}^{N}\left\|\phi_{i}\right\|_{\tau}^{2}$. Hence, we have $\bar{V}\left(t_{0}\right) \leq \mu \cdot\|\phi\|_{\tau}^{2}$. Thus, by (4.13), we get, for $t \in\left[t_{0}, t_{1}\right)$,

$$
V(e(t)) \leq \bar{V}\left(t_{0}\right) e^{-\sigma\left(t-t_{0}\right)} \leq \mu \cdot\|\phi\|_{\tau}^{2} e^{-\sigma\left(t-t_{0}\right)}=M^{0} \mu \cdot\|\phi\|_{\tau}^{2} e^{-\sigma\left(t-t_{0}\right)} .
$$

Thus, (4.15) holds for $k=1$.

Now, assume (4.15) holds for $k \leq m$, where $m>1$. Then, we show that (4.15) holds for $k=m+1$. By (4.14), (4.4), and the induction assumption, we have

$$
\begin{aligned}
V\left(e\left(t_{m}\right)\right) & \leq \beta_{m} V\left(e\left(t_{m}^{-}\right)\right) \leq \mu \beta_{m} M^{m-1}\|\phi\|_{\tau}^{2} e^{-\sigma\left(t_{m}-t_{0}\right)} \\
& \leq \mu M^{m}\|\phi\|_{\tau}^{2} e^{-\sigma\left(t_{m}-t_{0}\right)} .
\end{aligned}
$$

Hence, by condition (iii) and (4.17), for $k=m+1, t \in\left[t_{m}, t_{m+1}\right)$ we get

$$
\begin{aligned}
V(e(t)) & \leq \bar{V}\left(t_{m}\right) \cdot e^{-\sigma\left(t-t_{m}\right)}=\max \left\{\sup _{t_{m}-\tau \leq t<t_{m}}\{V(e(t))\}, V\left(e\left(t_{m}\right)\right)\right\} e^{-\sigma\left(t-t_{m}\right)} \\
& \leq \max \left\{\mu M^{m-1}\|\phi\|_{\tau}^{2} e^{-\sigma\left(t_{m}-\tau-t_{0}\right)}, \mu M^{m}\|\phi\|_{\tau}^{2} e^{-\sigma\left(t_{m}-t_{0}\right)}\right\} e^{-\sigma\left(t-t_{m}\right)} \\
& \leq \mu M^{m}\|\phi\|_{\tau}^{2} e^{-\sigma\left(t-t_{0}\right)} .
\end{aligned}
$$

Copyright (c) by SIAM. Unauthorized reproduction of this article is prohibited. 
Therefore, by the induction principle, we see that (4.15) holds for all $k \in N$.

Lastly, we show that

$$
\|e(t)\| \leq K\|\phi\|_{\tau} e^{-\alpha\left(t-t_{0}\right)}, \quad t \geq t_{0},
$$

where $\alpha=\frac{1}{2}\left\{\sigma-\frac{\ln M}{\delta \tau}\right\}>0, K=\sqrt{\frac{\mu}{\nu}}, \nu=\min _{1 \leq i \leq N}\left\{\nu_{i}\right\}$.

Since $\delta \tau \leq \Delta_{\text {inf }}=\inf _{k \in N}\left\{t_{k+1}-t_{k}\right\}$, we have $k \leq \frac{t_{k}-t_{0}}{\delta \tau}$, which implies $M^{k} \leq$ $e^{\frac{\ln M}{\delta \tau}\left(t_{k}-t_{0}\right)}$. Thus, for $t \in\left[t_{k}, t_{k+1}\right)$, we get

$$
\|e(t)\|^{2} \leq \frac{V(e(t))}{\nu} \leq \frac{\mu}{\nu}\|\phi\|_{\tau}^{2} M^{k-1} e^{-\sigma\left(t-t_{0}\right)} \leq \frac{\mu}{\nu}\|\phi\|_{\tau}^{2} e^{-\left(\sigma-\frac{\ln M}{\delta \tau}\right)\left(t-t_{0}\right)} .
$$

Hence, by (4.20), the error system (3.6) is RGES with decay rate $\alpha=\frac{1}{2}\left\{\sigma-\frac{\ln M}{\delta \tau}\right\}$. Thus, the CDN (3.1) can achieve GEIC with the given state $s(t)$ at rate $\alpha$.

Remark 4.1.

1. By (4.4), in condition (iii) of Theorem 4.1, it is permitted that the maximal impulsive gain $\beta \triangleq \sup _{k \in \mathbb{Z}}\left\{\beta_{k}\right\} \geq e^{\sigma \tau}>1$. This means that under the hybrid control scheme, the CDN not only can achieve GEIC but also is robust to some extent with respect to impulses existing in the control input signals. In this case, there is a requirement on minimal dwell time $\Delta_{\text {inf }}$ (see (4.4)) such that it has sufficient active time between two consecutive impulses to guarantee the GEIC property of the whole CDN. Moreover, if we choose the impulsive distributed controllers $\hat{u}_{i}(t, x, s)$ proposed (see Remark 3.2), we can derive a similar result. Here, the impulsive gain $\beta_{k}$ in Theorem 4.1 is chosen as $\beta_{k}=\max _{1 \leq i \leq N}\left\{\lambda_{\max }\left(P_{i}^{-1} \sum_{j=1}^{N} \beta_{j_{k}} P_{j}\right)\right\}$, where $\beta_{j_{k}}>0$ satisfies the following matrix inequality for $j=1,2, \ldots, N$ :

$$
\left(\begin{array}{cccc}
C_{j 1_{k}}^{T} P_{j} C_{j 1_{k}}-\beta_{j_{k}} P_{j} & C_{j 1_{k}}^{T} P_{j} C_{j 2_{k}} & \cdots & C_{j 1_{k}} P_{j} C_{j N_{k}} \\
C_{j 2_{k}}^{T} P_{j} C_{j 1_{k}} & C_{j 2_{k}}^{T} P_{j} C_{j 2_{k}}-\beta_{j_{k}} P_{j} & \cdots & C_{j 2_{k}}^{T} P_{j} C_{j 2_{k}} \\
\vdots & \vdots & \ddots & \vdots \\
C_{j N_{k}}^{T} P_{j} C_{j 1_{k}} & C_{j N_{k}}^{T} P_{j} C_{j 2_{k}} & \cdots & C_{j N_{k}}^{T} P_{j} C_{j N_{k}}-\beta_{j_{k}} P_{j}
\end{array}\right) \leq 0 .
$$

2. By condition (ii) of Theorem 4.1 and parameters $\alpha_{i}<0, i=1,2, \ldots, N$, LMIs (4.2) imply that positive definite matrices $P_{i}, i=1,2, \ldots, N$, satisfy

$$
A_{i_{0}}^{T} P_{i}+P_{i} A_{i_{0}}<0, \quad i=1,2, \ldots, N .
$$

A necessary condition for (4.22) to hold is that matrix $A_{i_{0}}$ must be stable, i.e., all the real parts of eigenvalues of $A_{i_{0}}$ are negative. For Hopfield neural networks (HNNs) and cell neural networks (see $[50,51,52]$ ), it is true. However, for some dynamical networks such as when the nodes in the network are chaotic systems, inequalities in (4.22), and thus condition (ii) of Theorem 4.1, cannot be satisfied. Hence, it is necessary to investigate the more general case, where $A_{i_{0}}$ is allowed to be unstable.

Theorem 4.2. Let Assumptions 3.1-3.3 be satisfied. Suppose $\Delta_{\text {sup }}<\infty$ and that there exist positive definite matrices $P_{i} \in \mathbb{R}^{n \times n}$ satisfying (4.1) and constants $\epsilon_{i j}>0$, $\varepsilon_{i}>0, \hat{\alpha}_{i}>0, i, j=1,2, \ldots, N$, such that 
(i) for $i=1,2, \ldots, N$, the following LMIs hold:

$$
\left(\begin{array}{cccccc}
\Psi_{i}\left(A_{i_{0}}\right)-\hat{\alpha}_{i} P_{i} & P_{i} B_{i 1_{0}} & \cdots & P_{i} B_{i N_{0}} & P_{i} E & F_{A_{i}}^{T} \\
B_{i 1_{0}}^{T} P_{i} & -\epsilon_{i 1} I & \cdots & 0 & 0 & F_{i 1}^{T} \\
\vdots & \vdots & \ddots & \vdots & \vdots & \vdots \\
B_{i N_{0}}^{T} P_{i} & 0 & \cdots & -\epsilon_{i N} I & 0 & F_{i N}^{T} \\
E^{T} P_{i} & 0 & \cdots & 0 & -\varepsilon_{i}^{-1} I & 0 \\
F_{A_{i}} & F_{i 1} & \cdots & F_{i N} & 0 & -\varepsilon_{i} I
\end{array}\right)<0
$$

where $\Psi_{i}\left(A_{i_{0}}\right)=P_{i} A_{i_{0}}+A_{i_{0}}^{T} P_{i}+2 L_{i} \sqrt{\frac{\mu_{i}}{\nu_{i}}} P_{i}+\sum_{j=1}^{N} M_{i j} \epsilon_{i j}^{-1}\left\|P_{i}\right\| I$;

(ii) for any $k \in \mathbb{Z}$,

$$
\beta_{k} \triangleq \max _{1 \leq i \leq N}\left\{\lambda_{\max }\left(P_{i}^{-1} C_{i_{k}}^{T} P_{i} C_{i_{k}}\right)\right\}<1 ;
$$

(iii) there exists a positive integer $m \geq 1$ such that $t_{k-m} \leq t_{k}-\tau<t_{k+1-m}$ for any $k \geq m, k \in \mathbb{Z}$, and the discrete system

$$
z(k+1)=J_{k}(m) z(k), \quad k \in \mathbb{Z},
$$

is GES with decay rate $\sigma>0$, where

$$
J_{k}(m) \triangleq\left(\begin{array}{cccccc}
0 & 1 & 0 & \cdots & 0 & 0 \\
0 & 0 & 1 & \cdots & 0 & 0 \\
\vdots & \vdots & \vdots & \cdots & \vdots & \vdots \\
0 & 0 & 0 & \cdots & 0 & 1 \\
\alpha_{k+1-m} & \alpha_{k+2-m} & \alpha_{k+3-m} & \cdots & \alpha_{k-1} & \tilde{\alpha}_{k-1}
\end{array}\right),
$$

where $\tilde{\alpha}_{k-1}=\beta_{k} e^{p \Delta_{k-1}}+\alpha_{k-1}, \alpha_{k-j}=p_{2} \Delta_{k-j} e^{p \Delta_{k-j}}, j=1,2, \ldots, m$, and $p=p_{1}+$ $p_{2}, p_{1}=\max _{1 \leq i \leq N}\left\{\hat{\alpha}_{i}\right\}, p_{2}=\max _{1 \leq i \leq N}\left\{\sum_{j=1}^{N} \frac{\hat{\epsilon}_{j i}}{v_{i}}\right\}$, where $\hat{\epsilon}_{i j}=\epsilon_{i j}+M_{i j} \epsilon_{i j}\left\|P_{i}\right\|$;

(iv) there exists a constant $\mathcal{T}_{0} \geq 0$ such that the average dwell time $\mathcal{T}_{a}$ satisfies

$$
S\left[t_{0}, t\right] \geq-\mathcal{T}_{0}+\frac{t-t_{0}}{\mathcal{T}_{a}}, \quad t \geq t_{0} .
$$

Then, the error system (3.6) is RGES with decay rate $\frac{\sigma}{2 \mathcal{T}_{a}}$, and hence $C D N(3.1)$ can achieve $G E I C$ with $s(t)$ at rate $\frac{\sigma}{2 T_{a}}$.

Proof. Let the Lyapunov-Krasovskii functional candidate $V$ be $V(e(t))=V_{1}(t)+$ $V_{2}(t)$, where for some positive constants $\lambda_{i j}>0, i, j=1,2, \ldots, N$,

$$
V_{1}(t)=\sum_{i=1}^{N} e_{i}^{T}(t) P_{i} e_{i}(t), \quad V_{2}(t)=\sum_{i=1}^{N} \sum_{j=1}^{N} \lambda_{i j} \int_{t-h_{j}}^{t} e_{i}^{T}(s) e_{i}(s) d s .
$$

Denote $\mu=\max _{1 \leq i \leq N}\left\{\mu_{i}\right\}, \nu=\min _{1 \leq i \leq N}\left\{\nu_{i}\right\}$. Then, for any $t \geq t_{0}-\tau$, we have

$$
\nu\|e(t)\|^{2} \leq V(e(t)) \leq \mu\|e(t)\|^{2}+\tau \sum_{i=1}^{N} \sum_{j=1}^{N} \lambda_{i j}\|e(t)\|_{\tau}^{2} .
$$

Similarly to the proof of Theorem 4.1, by (4.1) and condition (i), for $t \in\left[t_{k}, t_{k+1}\right.$ ), we have

$$
D^{+} V_{1}(t) \leq \max _{1 \leq i \leq N}\left\{\hat{\alpha}_{i}\right\} \sum_{i=1}^{N} e_{i}^{T} P_{i} e_{i}+\sum_{i=1}^{N} \sum_{j=1}^{N} \hat{\epsilon}_{j i} e_{i}^{T}\left(t-h_{j}\right) e_{i}\left(t-h_{j}\right),
$$

Copyright (c) by SIAM. Unauthorized reproduction of this article is prohibited. 
where $\hat{\epsilon}_{i j}=\epsilon_{i j}+M_{i j} \epsilon_{i j}\left\|P_{i}\right\|$. And for $t \in\left[t_{k}, t_{k+1}\right)$, we get

$$
\begin{aligned}
D^{+} V_{2}(t) \leq & \max _{1 \leq i \leq N}\left\{\sum_{j=1}^{N} \frac{\lambda_{i j}}{v_{i}}\right\} \sum_{i=1}^{N} e_{i}^{T}(t) P_{i} e_{i}(t) \\
& -\sum_{i=1}^{N} \sum_{j=1}^{N} \lambda_{i j} e_{i}^{T}\left(t-h_{j}\right) e_{i}\left(t-h_{j}\right) .
\end{aligned}
$$

Thus, letting $\lambda_{i j}=\hat{\epsilon}_{j i}$ for $i, j=1,2, \ldots, N$, for any $t \in\left[t_{k}, t_{k+1}\right)$, yields from (4.29)-

$$
D^{+} V(e(t))=D^{+} V_{1}(t)+D^{+} V_{2}(t) \leq\left(p_{1}+p_{2}\right) V_{1}(t) \leq p V(e(t)),
$$

where $p=p_{1}+p_{2}>0, p_{1}=\max _{1 \leq i \leq N}\left\{\hat{\alpha}_{i}\right\}$, and $p_{2}=\max _{1 \leq i \leq N}\left\{\sum_{j=1}^{N} \frac{\hat{\epsilon}_{j i}}{v_{i}}\right\}$.

Hence, by (4.31), we get that

$$
V(e(t)) \leq V\left(e\left(t_{k}\right)\right) e^{p\left(t-t_{k}\right)}, \quad t \in\left[t_{k}, t_{k+1}\right), k \in \mathbb{Z} .
$$

Moreover, when $t=t_{k}$, we obtain that

$$
V_{1}\left(t_{k}\right) \leq \beta_{k} V_{1}\left(t_{k}^{-}\right), \quad k \in \mathbb{Z} .
$$

By condition (iii), there exists $m \geq 1, m \in \mathbb{Z}$, such that $t_{k-m} \leq t_{k}-\tau<t_{k+1-m}$ for any $k \in \mathbb{Z}$. Then, for $t=t_{k}$, by the definition of $V_{2}(t)$, we get

$$
\begin{aligned}
V_{2}\left(t_{k}\right) & \leq p_{2} \int_{t_{k}-\tau}^{t_{k}} \sum_{i=1}^{N} e_{i}^{T}(s) P_{i} e_{i}^{T}(s) d s \leq p_{2} \int_{t_{k-m}}^{t_{k}} V_{1}(s) d s \\
& =p_{2} \sum_{j=1}^{m} \int_{t_{k-j}}^{t_{k-j+1}} V_{1}(s) d s=p_{2} \sum_{j=1}^{m} \Delta_{k-j} V_{1}\left(\hat{t}_{k-j+1}\right)
\end{aligned}
$$

for some $\hat{t}_{k-j+1} \in\left[t_{k-j}, t_{k-j+1}\right), j=1,2, \ldots, m$.

Then, by (4.32) and (4.34), we get

$$
V_{2}\left(t_{k}\right) \leq p_{2} \sum_{j=1}^{m} \Delta_{k-j} e^{p \Delta_{k-j}} V\left(e\left(t_{k-j}\right)\right) .
$$

Thus, it follows from (4.33) and (4.35) that

$$
\begin{aligned}
V\left(e\left(t_{k}\right)\right) & \leq\left(\beta_{k}+p_{2} \Delta_{k-1}\right) e^{p \Delta_{k-1}} V\left(e\left(t_{k-1}\right)\right)+p_{2} \sum_{j=2}^{m} \Delta_{k-j} e^{p \Delta_{k-j}} V\left(e\left(t_{k-j}\right)\right) \\
& \triangleq \tilde{\alpha}_{k-1} V\left(e\left(t_{k-1}\right)\right)+\sum_{j=1}^{m-1} \alpha_{k-j-1} V\left(e\left(t_{k-j-1}\right)\right),
\end{aligned}
$$

where $\tilde{\alpha}_{k-1}=\beta_{k} e^{p \Delta_{k-1}}+\alpha_{k-1}$ and $\alpha_{k-j}=p_{2} \Delta_{k-j} e^{p \Delta_{k-j}}, j=1,2, \ldots, m$.

For $k \in \mathbb{Z}$, let

$$
\left\{\begin{array}{l}
w_{1}(k)=V\left(e\left(t_{k+1}\right)\right) \\
w_{2}(k)=V\left(e\left(t_{k+2}\right)\right) \\
\vdots \\
w_{m}(k)=V\left(e\left(t_{k+m}\right)\right)
\end{array}\right.
$$

Copyright (c) by SIAM. Unauthorized reproduction of this article is prohibited. 
and $W(k)=\left(w_{1}(k), w_{2}(k), \ldots, w_{m}(k)\right)^{T}$. Then, by (4.36)-(4.37), it follows that

$$
\left(\begin{array}{c}
w_{1}(k+1-m) \\
w_{2}(k+1-m) \\
\vdots \\
w_{m}(k+1-m)
\end{array}\right) \leq J_{k}(m)\left(\begin{array}{c}
w_{1}(k-m) \\
w_{2}(k-m) \\
\vdots \\
w_{m}(k-m)
\end{array}\right),
$$

where $J_{k}(m)$ is defined by (4.26).

Thus, by (4.38), we get

$$
W(k-m+1) \leq J_{k}(m) W(k-m), \quad k \in \mathbb{Z} .
$$

Let the comparison system be

$$
\left\{\begin{array}{l}
z(k+1)=J_{k}(m) z(k), \quad k \geq m-1, \\
z(m-1)=W(-1) .
\end{array}\right.
$$

Then, by the comparison principle [45], we get

$$
W(k-m) \leq z(k), \quad k \geq m-1 .
$$

Thus, by condition (iii), i.e., the discrete system (4.25) is GES with decay rate $\sigma>0$, there exists constant $K>0$ such that

$$
\|W(k-m)\| \leq K e^{-\sigma(k-m+1)}\|W(-1)\|, \quad k \geq m-1, k \in \mathbb{Z},
$$

where $\|W(-1)\|^{2}=\sum_{j=0}^{m-1} V\left(e\left(t_{j}\right)\right)^{2}$ and $\|W(k-m)\|^{2}=\sum_{j=1}^{m} V\left(e\left(t_{j+k-m}\right)\right)^{2}$.

Let $\bar{\lambda}=\sum_{i=1}^{N} \sum_{j=1}^{N} \hat{\epsilon}_{j i}$; then, for $j=1,2, \ldots, m-1$, we get

$$
\begin{aligned}
V_{2}\left(t_{j}\right) & =\sum_{i=1}^{N} \sum_{k=1}^{N} \lambda_{i k} \int_{t_{j}-h_{k}}^{t_{j}} e_{i}^{T}(s) e_{i}(s) d s \leq \sum_{i=1}^{N} \sum_{k=1}^{N} \lambda_{i k} \int_{t_{0}-\tau}^{t_{j}} e_{i}^{T}(s) e_{i}(s) d s \\
& =\sum_{i=1}^{N} \sum_{k=1}^{N} \lambda_{i k}\left[\int_{t_{0}-\tau}^{t_{0}} e_{i}^{T}(s) e_{i}(s) d s+\sum_{l=0}^{j-1} \int_{t_{l}}^{t_{l+1}} e_{i}^{T}(s) e_{i}(s) d s\right] \\
& \leq \bar{\lambda} \tau\|\phi\|_{\tau}^{2}+p_{2} \sum_{l=0}^{j-1} V\left(e\left(t_{l}\right)\right) e^{p \Delta_{l-1}} .
\end{aligned}
$$

Thus, for $j=1,2, \ldots, m-1$, we get

$$
\begin{aligned}
V\left(e\left(t_{j}\right)\right) & \leq \bar{\lambda} \tau\|\phi\|_{\tau}^{2}+\beta_{j} e^{p \Delta_{j-1}} V\left(e\left(t_{j-1}\right)\right)+p_{2} \sum_{l=0}^{j-1} V\left(e\left(t_{l}\right)\right) e^{p \Delta_{l-1}} \\
& =\bar{\lambda} \tau\|\phi\|_{\tau}^{2}+\tilde{\alpha}_{j-1} V\left(e\left(t_{j-1}\right)\right)+\sum_{l=0}^{j-2} \alpha_{l} V\left(e\left(t_{l}\right)\right) .
\end{aligned}
$$

Moreover, it follows from (4.28) and $\lambda_{i j}=\hat{\epsilon}_{i j}$ that

$$
V\left(e\left(t_{0}\right)\right) \leq(\mu+\bar{\lambda} \tau)\|\phi\|_{\tau}^{2} .
$$

Thus, from (4.44)-(4.45), by the induction method, there exist positive constants $M_{j}$, which are dependent on $\tau, \bar{\lambda}, \beta_{j}, p_{2}, p, \Delta_{j}, j=1,2, \ldots, m-1$, such that

$$
V\left(e\left(t_{j}\right)\right) \leq M_{j}\|\phi\|_{\tau}^{2}, \quad j=1,2, \ldots, m-1 .
$$

Copyright (c) by SIAM. Unauthorized reproduction of this article is prohibited. 
Hence, we get

$$
\|W(-1)\|^{2}=\sum_{j=0}^{m-1} V\left(e\left(t_{j}\right)\right)^{2} \leq\left((\mu+\bar{\lambda} \tau)^{2}+\sum_{j=1}^{m-1} M_{j}^{2}\right)\|\phi\|_{\tau}^{4} .
$$

By (4.42) and (4.47), for any $k \in \mathbb{Z}$, it follows that

$$
V\left(e\left(t_{k}\right)\right) \leq\|W(k-m)\| \leq \sqrt{(\mu+\bar{\lambda} \tau)^{2}+\sum_{j=1}^{m-1} M_{j}^{2}} K e^{-\sigma(k-m+1)}\|\phi\|_{\tau}^{2} .
$$

Thus, by (4.28), (4.32), and (4.48), for any $t \in\left[t_{k}, t_{k+1}\right), k \in \mathbb{Z}$, we get

$$
\|e(t)\|^{2} \leq \frac{1}{\nu} V(e(t)) \leq \frac{\beta_{k}}{\nu} e^{p\left(t-t_{k}\right)} V\left(e\left(t_{k}\right)\right) \leq \tilde{K}^{2}\|\phi\|_{\tau}^{2} e^{-\sigma k},
$$

where $\tilde{K}^{2}=\frac{\sqrt{(\mu+\bar{\lambda} \tau)^{2}+\sum_{j=1}^{m-1} M_{j}^{2}} \cdot K e^{\sigma(m-1)+p \Delta_{\text {sup }}}}{\nu}$.

For any $t \in\left[t_{k}, t_{k+1}\right.$ ), we have $S\left[t_{0}, t\right]=k$, which yields by condition (iv) that $k \geq-\mathcal{T}_{0}+\frac{t-t_{0}}{\mathcal{T}_{a}}$ for any $t \in\left[t_{k}, t_{k+1}\right), k \in \mathbb{Z}$. Thus, by (4.49), we get

$$
\|e(t)\| \leq \tilde{K} e^{\frac{\sigma \mathcal{T}_{0}}{2}}\|\phi\|_{\tau} e^{-\frac{\sigma}{2 T_{a}}\left(t-t_{0}\right)}, \quad t \geq t_{0}
$$

which yields all the results of this theorem. The proof is thus complete.

Remark 4.2.

1. By condition (i) of Theorem 4.2 and parameters $\hat{\alpha}_{i}>0$, we get that matrices $A_{i_{0}}^{T} P_{i}+P_{i} A_{i_{0}}$ may be nonnegative definite, i.e., $A_{i_{0}}^{T} P_{i}+P_{i} A_{i_{0}} \geq 0, i=1,2, \ldots, N$. Thus, matrices $A_{i_{0}}, i=1,2, \ldots, N$, are allowed to be unstable. In this case, the impulsive control is key in stabilizing the error system and thus in the CDN achieving GEIC. It requires the impulsive gains $\beta_{k}<1, k \in \mathbb{Z}$, to offset the increment of $V$ led by the unstable dynamics during intervals $\left(t_{k}, t_{k+1}\right), k \in \mathbb{Z}$. Clearly, it should have enough numbers of impulse to enlarge the decrement of $V$ at these impulsive instances. Hence, there is a requirement on minimal impulse numbers to provide sufficient impulsive control signals. The inequality (4.27) gives the estimation of the minimal impulse times in time interval $\left[t_{0}, t\right], t \in \mathbb{R}_{+}$. Obviously, it also needs $\Delta_{\text {sup }}<\infty$; otherwise, from some time on, there is no impulsive control signal, and the error system is changed to an unstable continuous-time system.

2. It should be noted that the results of Theorems 4.1 and 4.2 are based on the solvability of LMIs in (4.2) and (4.23), respectively. For Theorem 4.1, as discussed in Remark 4.1.2, a necessity for the solvability for LMIs in (4.2) is that midpoint matrices $A_{i_{0}}, i=1,2, \ldots, N$, are Hurwitz. In addition, it also depends on how stable these midpoint matrices are and how big the matrix intervals $N\left[A_{i_{1}}, A_{i_{2}}\right], i=1,2, \ldots, N$, are as well. That is, the solvability of LMIs in (4.2) relates to the size of negative real parts of eigenvalues of midpoint matrices and the size of these matrix intervals. But for Theorem 4.2, there exists no solvability issue for LMIs in (4.23). Since for midpoint matrices $A_{i_{0}}$ (stable or unstable), and for any given symmetric matrices $Q_{i} \geq 0$, the matrix inequality $P_{i} A_{i_{0}}+A_{i_{0}}^{T} P_{i}+Q_{i}-\hat{\alpha}_{i} P_{i}<0$ is always solvable for some $P_{i}>0$ and some constants $\hat{\alpha}_{i}>0, i=1,2, \ldots, N$, and thus by the Schur complement theorem it is easy to see that LMIs in (4.23) are solvable.

In the following, by using Theorem 4.2, we give some simple GEIC criteria for CDN (3.1). 
Corollary 4.1. Let Assumptions 3.1-3.3 be satisfied. Suppose that $\tau \leq t_{k}-t_{k-1}$ for any $k \in \mathbb{Z}$, i.e., $m=1$ in Theorem 4.2 , and that there exist matrices $P_{i}>0$ and constants $\epsilon_{i j}>0, \varepsilon_{i}>0, \hat{\alpha}_{i}>0, i, j=1, \ldots, N$, such that (4.1) and (i), (ii), and (iv) of Theorem 4.2 hold, while condition (iii) of Theorem 4.2 is replaced by the following:

(iii*) There exists a positive constant $\sigma$ satisfying $\sigma>0$ such that

$$
\ln \left(\beta_{k}+p_{2} \tau\right)+p \Delta_{k-1} \leq-\sigma, \quad k \geq 1, k \in \mathbb{Z}
$$

where $\beta_{k}=\max _{1 \leq i \leq N}\left\{\lambda_{\max }\left(P_{i}^{-1} C_{i_{k}}^{T} P_{i} C_{i_{k}}\right)\right\}<1$ and $p=p_{1}+p_{2}, p_{1}=\max _{1 \leq i \leq N}$ $\left\{\hat{\alpha}_{i}\right\}, p_{2}=\max _{1 \leq i \leq N}\left\{\frac{1}{\nu_{i}} \sum_{j=1}^{N} \hat{\epsilon}_{j i}\right\}, \hat{\epsilon}_{i j}=\epsilon_{i j}+M_{i j} \epsilon_{i j}\left\|P_{i}\right\|$.

Then, system (3.6) is RGES with decay rate $\frac{\sigma}{2 \mathcal{T}_{a}}$, and thus $C D N$ (3.1) achieves GEIC with $s(t)$.

Proof. Take the same Lyapunov-Krasovskii functional $V$ as in Theorem 4.2, that is, $V(e(t))=V_{1}(t)+V_{2}(t)$ as follows:

$$
V_{1}(t)=\sum_{i=1}^{N} e_{i}^{T}(t) P_{i} e_{i}(t), \quad V_{2}(t)=\sum_{i=1}^{N} \sum_{j=1}^{N} \lambda_{i j} \int_{t-h_{j}}^{t} e_{i}^{T}(s) e_{i}(s) d s
$$

where $\lambda_{i j}=\hat{\epsilon}_{j i}$ for $i, j=1,2, \ldots, N$. By the proof of Theorem 4.2, we obtain that (4.28)-(4.33) hold. For $t=t_{k}$, by the definition of $V_{2}(t)$, we get

$$
V_{2}\left(t_{k}\right) \leq p_{2} \int_{t_{k}-\tau}^{t_{k}} \sum_{i=1}^{N} e_{i}^{T}(s) P_{i} e_{i}^{T}(s) d s=p_{2} \tau V_{1}\left(t_{k}+\theta_{k}\right)
$$

for some $\theta_{k} \in[-\tau, 0]$. Thus, it follows from $\tau \leq t_{k}-t_{k-1}$ and (4.32), (4.33), and (4.52) that

$$
\begin{aligned}
V\left(e\left(t_{k}\right)\right) & \leq \beta_{k} V_{1}\left(t_{k}^{-}\right)+p_{2} \tau V_{1}\left(t_{k}+\theta_{k}\right) \\
& \leq \beta_{k} V\left(e\left(t_{k-1}\right)\right) e^{p\left(t_{k}-t_{k-1}\right)}+p_{2} \tau V\left(e\left(t_{k-1}\right)\right) e^{p\left(t_{k}+\theta_{k}-t_{k-1}\right)} \\
& \leq e^{\ln \left(\beta_{k}+p_{2} \tau\right)+p\left(t_{k}-t_{k-1}\right)} V\left(e\left(t_{k-1}\right)\right), \quad k \in \mathbb{Z} .
\end{aligned}
$$

From condition (iii*) and (4.53), we get that for any $k \in \mathbb{Z}$,

$$
V\left(e\left(t_{k}\right)\right) \leq e^{-\sigma} V\left(e\left(t_{k-1}\right)\right) \leq \cdots \leq e^{-\sigma k} V\left(e\left(t_{0}\right)\right) .
$$

Thus, for any $t \in\left[t_{k}, t_{k+1}\right), k \in \mathbb{Z}$, by (4.54), condition (iii*), and inequality (4.27), we have

$$
V(e(t)) \leq e^{p\left(t-t_{k}\right)} e^{\sigma \mathcal{T}_{0}-\sigma\left(t_{k}-t_{0}\right)} V\left(e\left(t_{0}\right)\right) \leq e^{\sigma \mathcal{T}_{0}+\left|p-\frac{\sigma}{T_{a}}\right|} e^{-\frac{\sigma}{T_{a}}\left(t-t_{0}\right)} V\left(e\left(t_{0}\right)\right)
$$

By (4.28) and (4.55), for any initial condition $e(s)=\phi(s), s \in[-\tau, 0]$, and any $t \in\left[t_{k}, t_{k+1}\right), k \in \mathbb{Z}$, we get

$$
\|e(t)\| \leq \sqrt{\frac{\mu+\tau \sum_{i=1}^{N} \sum_{j=1}^{N} \epsilon_{j i}}{\nu}} \cdot \sqrt{e^{\sigma \mathcal{T}_{0}+\left|p-\frac{\sigma}{T_{a}}\right|}} \cdot\|\phi\|_{\tau} e^{-\frac{\sigma}{2 T_{a}}\left(t-t_{0}\right)} .
$$

Hence, it follows from (4.56) that all statements of this corollary are true.

Copyright $\odot$ by SIAM. Unauthorized reproduction of this article is prohibited. 
Remark 4.3.

1. From Corollary 4.1, if $\tau \leq t_{k}-t_{k-1}$ for any $k \in \mathbb{Z}$, we get estimations of maximal time-delay $\tau^{*}$ and maximal dwell time $\Delta_{\text {sup }}$ between two consecutive impulses as

$$
\tau^{*} \leq \sup _{k \geq 1, k \in \mathbb{Z}}\left\{\frac{e^{-p \Delta_{k-1}-\sigma}-\beta_{k}}{p}\right\}, \quad \Delta_{\text {sup }} \leq \sup _{k \geq 1, k \in \mathbb{Z}}\left\{\frac{-\sigma-\ln \left(\beta_{k}+p_{2} \tau\right)}{p}\right\} .
$$

2. From Corollary 4.1, one can see how robust these impulse control signals are with respect to the unstable midpoint matrices $A_{i_{0}}$ and the uncertainties in matrices $A_{i}, i=1,2, \ldots, N$. The parameter $p=p_{1}+p_{2}$, where $p_{1}$ is from unstable $A_{i_{0}}$, and $p_{2}$ is from uncertainties in matrices $A_{i}, i=1,2, \ldots, N$, reflects the instability level in (3.6). By (4.51), for smaller $\tau$, if we take the impulsive gains $\beta_{k}$ as

$$
0<\beta_{k}<e^{-p \Delta_{k-1}-\sigma}-p_{2} \tau, \quad k \in \mathbb{Z},
$$

then system (3.6) is RGES, and thus CDN (3.1) achieves GEIC at rate $\frac{\sigma}{2 \mathcal{T}_{a}}$.

Corollary 4.2. Assume all conditions of Theorem 4.2 hold except condition (iii), which is replaced by the following:

(iii**) There exists a positive integer $m>1$ such that $t_{k-m} \leq t_{k}-\tau<t_{k+1-m}$ for any $k \geq m, k \in \mathbb{Z}$, and the matrix $J(m)$ satisfies the spectral radius condition for some $\sigma>0$,

$$
\rho(J(m))<e^{-\sigma}
$$

where

$$
J(m) \triangleq\left(\begin{array}{cccccc}
0 & 1 & 0 & \cdots & 0 & 0 \\
0 & 0 & 1 & \cdots & 0 & 0 \\
\vdots & \vdots & \vdots & \cdots & \vdots & \vdots \\
0 & 0 & 0 & \cdots & 0 & 1 \\
a & a & a & \cdots & a & a+b
\end{array}\right),
$$

and $a=p_{2} \Delta_{\text {sup }} e^{p \Delta_{\text {sup }}}, b=\beta e^{p \Delta_{\text {sup }}}$, and $\beta=\sup _{k \in \mathbb{Z}}\left\{\lambda_{\max }\left(P_{i}^{-1} C_{i_{k}}^{T} P_{i} C_{i_{k}}\right)\right\}, p=p_{1}+$ $p_{2}, p_{1}=\max _{1 \leq i \leq N}\left\{\hat{\alpha}_{i}\right\}, p_{2}=\max _{1 \leq i \leq N}\left\{\frac{1}{\nu_{i}} \sum_{j=1}^{N} \hat{\epsilon}_{j i}\right\}$, where $\hat{\epsilon}_{i j}=\epsilon_{i j}+M_{i j} \epsilon_{i j}\left\|P_{i}\right\|$.

Then, system (3.6) is RGES with decay rate $\frac{\sigma}{2 \mathcal{T}_{a}}$, and hence $C D N$ (3.1) achieves GEIC at rate $\frac{\sigma}{2 \mathcal{T}_{a}}$.

Proof. In (4.36) of Theorem 4.2, since $\alpha_{k-m+j} \leq a(j=1,2, \ldots, m-1)$, and $\tilde{\alpha}_{k} \leq a+b$, then, by $(4.36)$, we get

$$
V\left(e\left(t_{k}\right)\right) \leq(a+b) V\left(e\left(t_{k-1}\right)\right)+a \sum_{j=1}^{m-1} V\left(e\left(t_{k-j+1}\right)\right) .
$$

Thus, we derive the comparison system

$$
z(k+1)=J(m) z(k), \quad k \geq m-1, k \in \mathbb{Z} .
$$

For the discrete system (4.62), it is well known that (4.62) is GES if and only if the spectral radius of matrix $J(m)$ satisfies $\rho(J(m))<1$. Thus, from $\rho(J(m))<e^{-\sigma}$ and by the similar proof of Theorem 4.2, we conclude that this corollary is true.

Corollary 4.3. Assume that all conditions of Theorem 4.2 hold except condition (iii), which is replaced by the following: 
(iii***) There exists a positive integer $m>1$ such that $t_{k-m} \leq t_{k}-\tau<t_{k+1-m}$ for any $k \geq m, k \in \mathbb{Z}$, and there exists some positive constant $0<\gamma<1$ such that every root $\lambda_{j}(j=1,2, \ldots, m)$ of the characteristic polynomial

$$
F_{k}(\lambda) \triangleq \lambda^{m}-\tilde{\alpha}_{k-1} \lambda^{m-1}-\alpha_{k-1} \lambda^{m-2}-\cdots-\alpha_{k+2-m} \lambda-\alpha_{k+1-m}
$$

satisfies that $\left|\lambda_{j}\right| \leq \gamma<1, j=1,2, \ldots, m$, where $\tilde{\alpha}_{k-1}, \alpha_{j}, j=k-1, k-2, \ldots, k+$ $1-m$, are the same as in Theorem 4.2 .

Then system (3.6) is $R G A S$, and hence $C D N(3.1)$ can achieve GIC with the given state $s(t)$.

Proof. It is easy to get that $F_{k}(\lambda)$ is the characteristic polynomial of matrix $J_{k}(m)$, i.e.,

$$
F_{k}(\lambda)=\left|\lambda I-J_{k}(m)\right| .
$$

Then, every root of $\lambda_{j}$ of $F_{k}(\lambda)$ is the characteristic root of $J_{k}(m)$. Hence, if every root $\lambda_{j}(j=1,2, \ldots, m)$ lies strictly in the unit circle and satisfies $\left|\lambda_{j}\right| \leq e^{-\sigma}<1$ for some $\sigma>0$, then the spectral radius of every matrix $J_{k}(m)$ satisfies $\rho\left(J_{k}(m)\right) \leq \gamma<1$. Thus, the comparison system $z(k+1)=J_{k}(m) z(k)$ is GAS, and hence we conclude that system (3.6) is RGAS, and thus CDN (3.1) achieves GIC with $s(t)$.

5. Impulsive consensus control design. In this section, by using Theorem 4.2, Corollaries 4.1-4.3, and Remark 4.3, we design impulsive control gain matrices $\left\{C_{i_{k}}, k \in\right.$ $\mathbb{Z}\}$ for the CDN (3.1) such that GEIC can be achieved by the impulsive consensus control.

TheOrem 5.1. Let Assumptions 3.1-3.3 be satisfied and let $\Delta_{\text {sup }}<\infty$. Assume that (4.1) and conditions (i) and (iv) of Theorem 4.2 still hold, while (ii)-(iii) are changed to (ii')-(iii') as follows:

(ii') There exist constants $0<\tilde{\beta}_{i}<1, i=1,2, \ldots, N$, such that the following LMIs hold:

$$
\left(\begin{array}{ccc}
\Omega_{1_{i}} & \Omega_{2_{i}} & Y_{i}^{T} \\
\Omega_{2_{i}}^{T} & -I & 0 \\
Y_{i} & 0 & -P_{i}
\end{array}\right) \leq 0, \quad i=1,2, \ldots, N
$$

where $\Omega_{1_{i}}=P_{i}+Y_{i}^{T}+Y_{i}-\tilde{\beta}_{i} P_{i}, \Omega_{2_{i}}=P_{i} E+Y_{i}^{T} E, i=1,2, \ldots, N$;

(iii') constants $\beta_{k} \triangleq \max _{1 \leq i \leq N}\left\{\tilde{\beta}_{i}\right\}, k \in \mathbb{Z}$, satisfy condition (ii) of Theorem 4.2 .

Then, under impulsive control gain matrices $\left\{C_{i_{k}}=I+P_{i}^{-1} Y_{i}, i=1,2, \ldots, N, k \in\right.$ $\mathbb{Z}\}$, the error system (3.6) is RGES with decay rate $\frac{\sigma}{2 \mathcal{T}_{a}}$, and hence the $C D N(3.1)$ achieves $G E I C$ with $s(t)$ at rate $\frac{\sigma}{2 \mathcal{T}_{a}}$.

Proof. Let $C_{i_{k}}=I+P_{i}^{-1} Y_{i}$; then, by using the Schur complement theorem, the LMI (5.1) is equivalent to

$$
C_{i_{k}}^{T} P_{i} C_{i_{k}}-\tilde{\beta}_{i} P_{i}+\Omega_{2}^{T} \Omega_{2} \leq 0
$$

which yields that $C_{i_{k}}^{T} P_{i} C_{i_{k}}-\tilde{\beta}_{i} P_{i} \leq 0$. Thus, we get that

$$
V_{1}\left(t_{k}\right)=\sum_{i=1}^{N} e_{i}^{T}\left(t_{k}^{-}\right) C_{i_{k}}^{T} P_{i} C_{i_{k}} e_{i}\left(t_{k}^{-}\right) \leq \sum_{i=1}^{N} \tilde{\beta}_{i} V_{1}\left(t_{k}^{-}\right) \leq \beta_{k} V_{1}\left(t_{k}^{-}\right), \quad k \in \mathbb{Z}
$$

It is not hard to see that the result of this theorem can be derived by using Theorem 4.2. The proof is thus complete. 
Corollary 5.1. Assume that (4.1) and conditions (i) and (iv) of Theorem 4.2 and condition (ii') of Theorem 5.1 hold. Then, under impulsive control gain matrices $\left\{C_{i_{k}}=I+P_{i}^{-1} Y_{i}, i=1,2, \ldots, N, k \in \mathbb{Z}\right\}$, condition (iii*) of Corollary 4.1 or (iii**) of Corollary 4.2 implies that $C D N(3.1)$ can achieve GEIC at rate $\frac{\sigma}{2 \mathcal{T}_{a}}$, where $\mathcal{T}_{a}$ satisfies (4.27), and (iii***) of Corollary 4.3 implies that the error system (3.6) is RGAS, and thus $C D N(3.1)$ can achieve GIC.

Proof. The results can be derived from Theorems 4.2 and 5.1 and Corollaries 4.14.3 .

6. Examples and numerical simulations. In this section, two representative examples are given for illustration.

Example 6.1. Consider CDN (3.1), where $x_{i}=\left(x_{i 1}, x_{i 2}, x_{i 3}\right)^{T}, i=1,2, \ldots, 10$, $f_{i}\left(t, x_{i}\right)=A_{i} x_{i}+\tilde{f}_{i}\left(t, x_{i}\right)$, in which $A_{i} \in N[\underline{A}, \bar{A}]$, where

$$
\underline{A}=\left(\begin{array}{ccc}
0.3570 & -13.2633 & -0.6431 \\
11.4195 & -0.1531 & -0.8700 \\
-0.2161 & -0.2204 & -0.0191
\end{array}\right), \quad \bar{A}=\left(\begin{array}{ccc}
1.3570 & -12.2633 & 0.3569 \\
12.4195 & 0.8469 & 0.1300 \\
0.7839 & 0.7796 & 0.9809
\end{array}\right) \text {, }
$$

$\tilde{f}_{i}\left(t, x_{i}\right)=\left(\sin t^{2},-\sin x_{i 2}-\cos t, \sin x_{i 3}+2 \sin (t-1)\right)^{T}$, and $g_{i}\left(x_{1}\left(t-h_{i}\right), \ldots, x_{N}(t-\right.$ $\left.\left.h_{i}\right)\right)=\sum_{j=1}^{N} B_{i j} x_{j}\left(t-h_{i}\right)=B_{i i} x_{i}\left(t-h_{i}\right)+B_{i, i+1} x_{i+1}\left(t-h_{i}\right)+B_{i, i+2} x_{i+2}\left(t-h_{i}\right)$, where $B_{i j}=B_{i j_{1}}=B_{i j_{2}}, i, j=1,2, \ldots, N$, and

$$
B_{i i}=\left(\begin{array}{ccc}
0.5 & 0.5 & 0 \\
0 & 0.5 & 0.2 \\
0 & 0 & -0.5
\end{array}\right), \quad B_{i, i+1}=\left(\begin{array}{ccc}
-1.0 & -0.3 & 0 \\
0 & 0.25 & -0.1 \\
0 & 0 & 1.0
\end{array}\right), \quad B_{i, i+2}=\left(\begin{array}{ccc}
0.5 & -0.2 & 0 \\
0 & -0.75 & -0.1 \\
0 & 0 & -0.5
\end{array}\right)
$$

while $B_{N-1, N+1}=B_{N-1,1}, B_{N-1, N+2}=N_{N-1,2}, B_{N, N+1}=B_{N 1}, B_{N, N+2}=B_{N 2}$.

The goal function $s(t)$ is $s(t)=\left(s_{1}(t), s_{2}(t), s_{3}(t)\right)^{T}=\left(\sin t, 2, e^{-2 t}\right)^{T}$.

In the following, we investigate the consensus between CDN (3.1) and the goal $s(t)$. It is easy to get

$$
A_{i_{0}}=\left(\begin{array}{ccc}
0.8570 & -12.7633 & -0.1431 \\
11.9195 & 0.3469 & -0.3700 \\
0.2839 & 0.2796 & 0.4809
\end{array}\right), \quad E=\left(\begin{array}{ccccccccc}
1 & 1 & 1 & 0 & 0 & 0 & 0 & 0 & 0 \\
0 & 0 & 0 & 1 & 1 & 1 & 0 & 0 & 0 \\
0 & 0 & 0 & 0 & 0 & 0 & 1 & 1 & 1
\end{array}\right) \text {, }
$$

and $F^{T}=E$.

Letting $\epsilon_{i j}=1, \varepsilon_{i}=1, \nu_{i}=1, \mu_{i}=2, \Delta_{k}=0.1, h_{i}=0.02, i=1,2, \ldots, 10, k \in \mathbb{Z}$, and solving the LMIs in (ii) of Theorem 4.2 , for $i=1,2, \ldots, 10$, we get $\hat{\alpha}_{i}=0.12$ and

$$
P_{i}=\left(\begin{array}{ccc}
1.0650 & -0.0364 & 0.0005 \\
-0.0364 & 1.0811 & -0.0135 \\
0.0005 & -0.0135 & 1.0567
\end{array}\right)
$$

Thus, we have $p_{1}=\max _{1 \leq i \leq 10}\left\{\alpha_{i}\right\}=0.12, p_{2}=\max _{1 \leq i \leq 10}\left\{\frac{1}{\nu_{i}} \sum_{j=1}^{10} \epsilon_{j i}\right\}=10$, and letting $\sigma=0.1160$, then, by (4.51), we obtain $\beta_{k}<0.12154$. Thus, the conditions of Corollary 4.1 are satisfied, and the error system is RGES with decay rate 0.5800. Hence, the network can achieve GEIC with $s(t)$ at rate 0.5800 .

In simulation, without loss of generality, initial conditions were set as $x_{i}(t)=0$ whenever $t<0$, and $x_{i}(0) \neq 0, i=1,2, \ldots, N$. The matrices $A_{i} \in N[\underline{A}, \bar{A}]$ are set as $A_{1}=A_{2}=A_{3}=\tilde{A}_{1}, A_{4}=A_{5}=A_{6}=\tilde{A}_{2}, A_{7}=A_{8}=\tilde{A}_{3}$, and $A_{9}=A_{10}=\tilde{A}_{4}$, where $\tilde{A}_{2}=A_{i_{0}}, \tilde{A}_{3}=\underline{A}, \tilde{A}_{4}=\bar{A}$, and

$$
\tilde{A}_{1}=\underline{A}+\operatorname{Rand}(3,3)=\left(\begin{array}{ccc}
-8.4496 & -11.7216 & 0.7353 \\
13.1017 & -9.0022 & 0.9900 \\
1.0867 & 1.4775 & -8.1654
\end{array}\right) \text {, }
$$

Copyright (c) by SIAM. Unauthorized reproduction of this article is prohibited. 


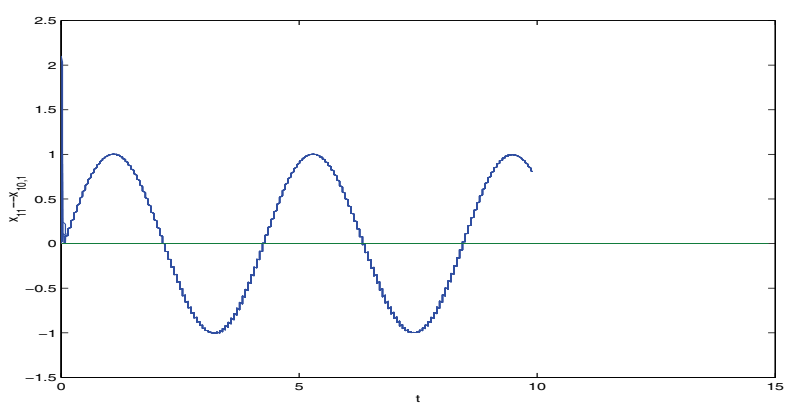

FIG. 6.1. Consensus properties of $x_{k 1}(t), k=1,2, \ldots, 10$, with $s_{1}=\sin (t)$.

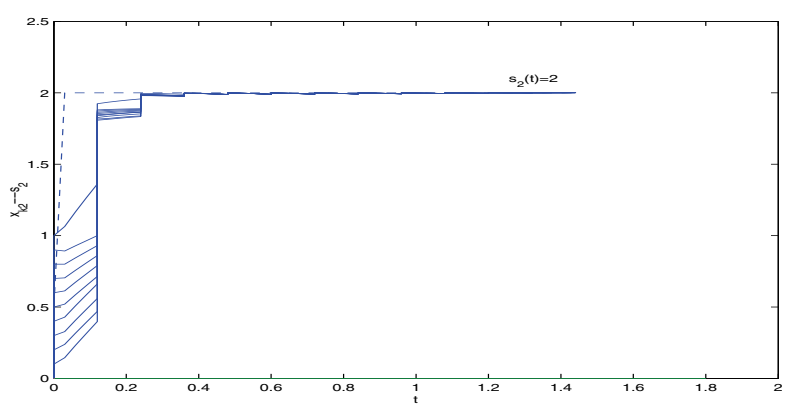

FIG. 6.2. Consensus properties of $x_{k 2}(t), k=1,2, \ldots, 10$, and the goal $s_{2}(t)$.

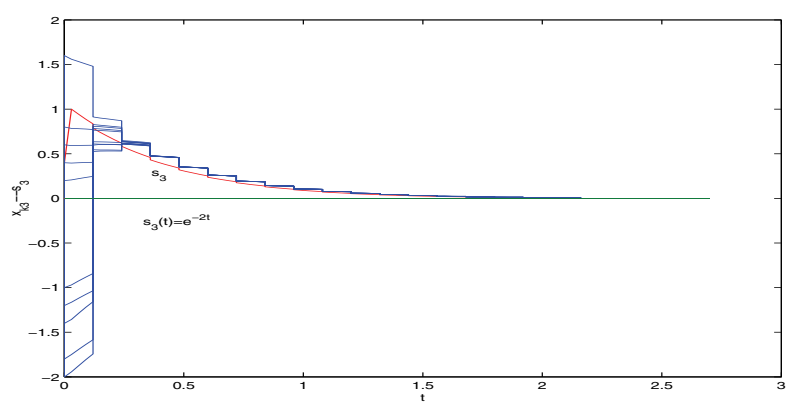

FIG. 6.3. Consensus properties of $x_{k 3}(t), k=1,2, \ldots, 10$, and the goal $s_{3}(t)$.

where matrix $\operatorname{Rand}(3,3)=\left(r_{i j}\right)$ is a $3 \times 3$ random matrix with entries $r_{i j}$ satisfying $0 \leq r_{i j} \leq 1$. From $\beta_{k}<0.12154$, in simulation, under Theorem 5.1, we design the impulsive matrices $C_{i_{k}}$ as $C_{i_{k}}=0.12 I$.

In Figures 6.1-6.3, one can see the consensus properties of the goal coordinate $s_{j}$ of $s(t)$ and substates $x_{i j}, i=1,2, \ldots, 10, j=1,2,3$, of the CDN.

Example 6.2. Use the chaotic Colpitts' oscillator as nodes of CDN. The Colpitts' oscillator is described by

$$
\dot{y}=A_{0} y+\varphi(y),
$$

Copyright $@$ by SIAM. Unauthorized reproduction of this article is prohibited. 
where

$$
y=\left(\begin{array}{l}
y_{1} \\
y_{2} \\
y_{3}
\end{array}\right), \quad A_{0}=\left(\begin{array}{ccc}
0 & \alpha & 0 \\
-\sigma & -\gamma \sigma & -\sigma \\
a_{1} \beta & \beta & 0
\end{array}\right), \quad \varphi(y)=\left(0,0, a_{3} \beta y_{1}^{3}\right)^{T},
$$

in which $\alpha, \beta, \sigma, a_{1}, \gamma, a_{3} \in R$. It is known that with parameters $\alpha=2.4, \beta=$ $2.2, \sigma=1, \gamma=0.252, a_{1}=1$, and $a_{3}=-0.2$, the oscillator (6.1) is chaotic.

Suppose that the CDN (3.1) with nonidentical nodes and time-delays is given by

$$
\dot{x}_{i}=A_{i} x_{i}+\varphi\left(x_{i}\right)+\sum_{j=1}^{N} B_{i j} x_{j}\left(t-h_{i}\right), \quad i=1,2, \ldots, N=10,
$$

where $x_{i}=\left(x_{i 1}, x_{i 2}, x_{i 3}\right)^{T}, B_{i j}(i, j=1,2, \ldots, N)$, are the same as those in Example 6.1 , and $A_{i} \in N[\underline{A}, \bar{A}]$ with

$$
\underline{A}=\left(\begin{array}{ccc}
-0.5 & \alpha-0.5 & -0.5 \\
-\sigma-0.5 & -\gamma \sigma-0.5 & -\sigma-0.5 \\
a_{1} \beta-0.5 & \beta-0.5 & -0.5
\end{array}\right), \quad \bar{A}=\left(\begin{array}{ccc}
0.5 & \alpha+0.5 & 0.5 \\
-\sigma+0.5 & -\gamma \sigma+0.5 & -\sigma+0.5 \\
a_{1} \beta+0.5 & \beta+0.5 & 0.5
\end{array}\right) .
$$

Suppose the goal vector function $s(t)$ is the solution of the Lorenz system

$$
\dot{s}=L s+\tilde{\varphi}(s),
$$

where

$$
s=\left(\begin{array}{l}
s_{1} \\
s_{2} \\
s_{3}
\end{array}\right), \quad L=\left(\begin{array}{ccc}
-b_{1} & b_{1} & 0 \\
b_{2} & -1 & 0 \\
0 & 0 & -b_{3}
\end{array}\right), \quad \varphi(s)=\left(0,-s_{1} s_{3}, s_{1} s_{2}\right)^{T},
$$

in which $b_{1}, b_{2}, b_{3} \in R$. It is well known that under $b_{1}=10, b_{2}=28, b_{3}=\frac{8}{3}$, Lorenz system (6.3) is chaotic.

It is easy to show for any matrix $A \in N[\underline{A}, \bar{A}], A$ is not a stable matrix. Hence, condition (i) of Theorem 4.1 is not satisfied. In the following, by using Theorem 4.2, we design the impulsive controllers $\left\{u_{i_{k}}, t_{k}\right\}=\left\{K_{i_{k}} x_{i}, t_{k}\right\}$ such that

$$
\left\{\begin{array}{l}
\dot{x}_{i}=A_{i} x_{i}+\varphi\left(x_{i}\right)+\sum_{j=1}^{N} B_{i j} x_{j}\left(t-h_{i}\right)+\dot{s}-\left[A_{i} s+\varphi(s)\right], t \in\left[t_{k}, t_{k+1}\right), \\
\Delta x_{i}(t)=u_{i_{k}}(t)=K_{i_{k}}\left(x_{i}(t)-s(t)\right), t=t_{k}, k \in \mathbb{Z}
\end{array}\right.
$$

can achieve GEIC.

By [44], we get that $\left\|\frac{\partial \varphi(y)}{\partial y}\right\| \leq 5.28$, which implies that $L_{i}=5.28, i=1, \ldots, 10$. By choosing $\epsilon_{i j}=1, \epsilon_{i}=1, \nu_{i}=1, \mu_{i}=2$, and solving the LMIs in (4.23) of Theorem 4.2 , for $i=1,2, \ldots, 10$, we get $\hat{\alpha}_{i}=6, p_{1}=6, p_{2}=10$, and

$$
P_{i}=\left(\begin{array}{lll}
1.5437 & 0.3646 & 0.1371 \\
0.3646 & 1.4298 & 0.1290 \\
0.1371 & 0.1290 & 1.1520
\end{array}\right)
$$

Then, by solving the LMIs in (4.23) of Theorem 5.1, we get that $\beta_{k}=0.01$, and

$$
Y_{i}=\left(\begin{array}{ccc}
-1.5328 & -0.3546 & -0.1330 \\
-0.3546 & -1.4215 & -0.1264 \\
-0.1330 & -0.1264 & -1.1502
\end{array}\right), \quad K_{i_{k}}-I=P_{i}^{-1} Y_{i}=\left(\begin{array}{ccc}
-0.9944 & 0.0054 & 0.0024 \\
0.0054 & -0.9957 & 0.0012 \\
0.0023 & 0.0011 & -1.0004
\end{array}\right) .
$$




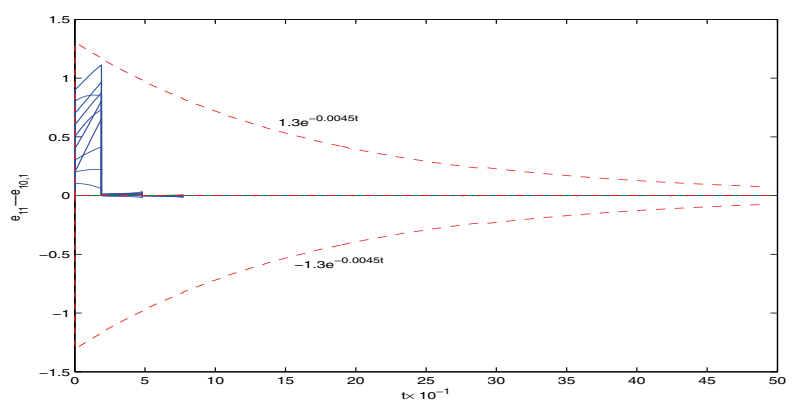

FIG. 6.4. Exponential stability properties of errors $e_{k 1}(t), k=1,2, \ldots, 10$.

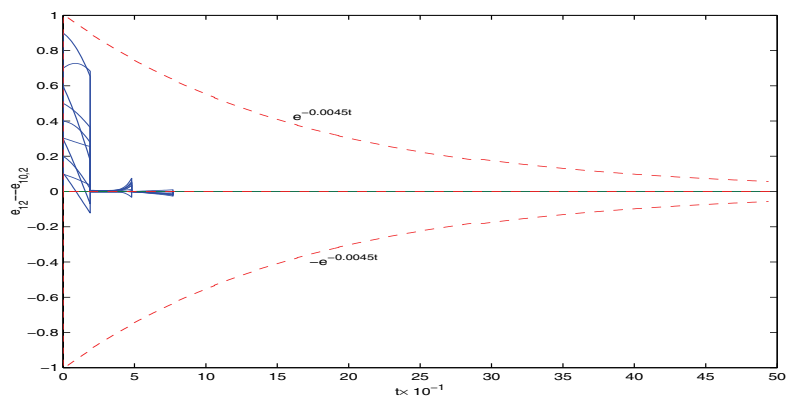

FIG. 6.5. Exponential stability properties of errors $e_{k 2}(t), k=1,2, \ldots, 10$.

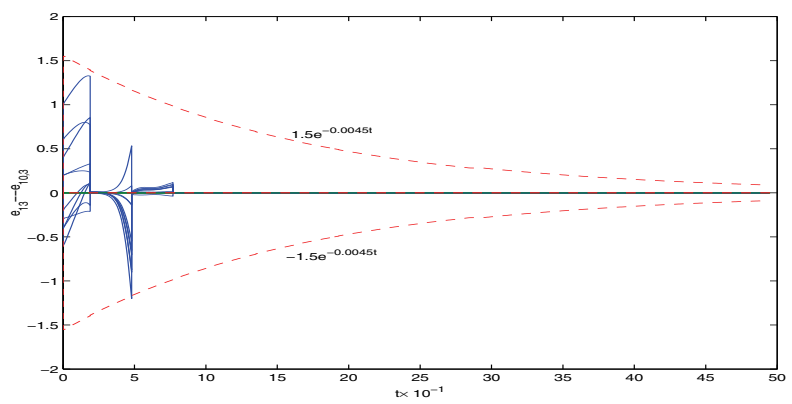

FIG. 6.6. Exponential stability properties of errors $e_{k 3}(t), k=1,2, \ldots, 10$.

Moreover, there exists $\sigma=0.001$ such that (4.51) in Corollary 4.1 holds. By Remark 4.3, we get $\tau^{*}<0.0619$ and $\Delta_{\text {sup }} \leq 0.1107$.

Thus, we can design the impulsive controllers $\left\{K_{i_{k}} x_{i}, t_{k}\right\}$ as follows.

Case 1. If $\tau \leq \Delta_{k}, k \in \mathbb{Z}$, then let $t_{0}=0, \Delta_{k}=0.1, k \in \mathbb{Z}$, and $K_{i_{k}}, i=$ $1,2, \ldots, 10$, be chosen as above. Therefore, by Theorem 5.1 , the impulsive controllers $\left\{K_{i_{k}} e_{i}, t_{k}\right\}$ can achieve GEIC between the CDN and $s(t)$. And the consensus rate is 0.005 .

Case 2. If there is $m>1$ such that $t_{k-m} \leq t_{k}-\tau<t_{k+1-m}, k \in \mathbb{Z}$, then, by Corollary 4.2, we design the impulsive controllers. For example, let $h_{i}=0.05$ and $m=2$; then by Corollary 4.2 , the impulsive instances can be set as $t_{0}=0, \Delta_{k}=$ $0.03, k \in \mathbb{Z}$. Therefore, by Corollary $4.2,\left\{K_{i_{k}} e_{i}, t_{k}\right\}$ can achieve GEIC between the $\mathrm{CDN}$ and $s(t)$. And the consensus rate is 0.0045 .

Copyright (c) by SIAM. Unauthorized reproduction of this article is prohibited. 


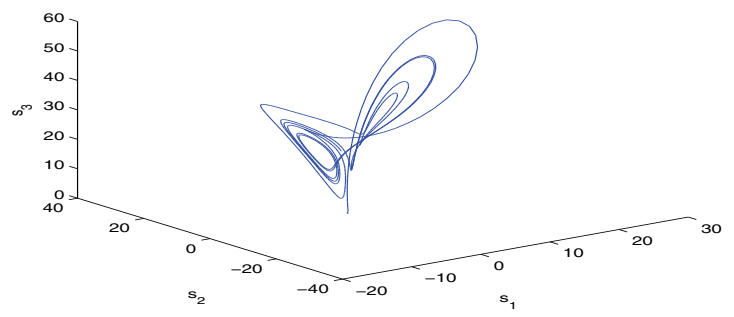

FIG. 6.7. The phase figure of the Lorenz system.

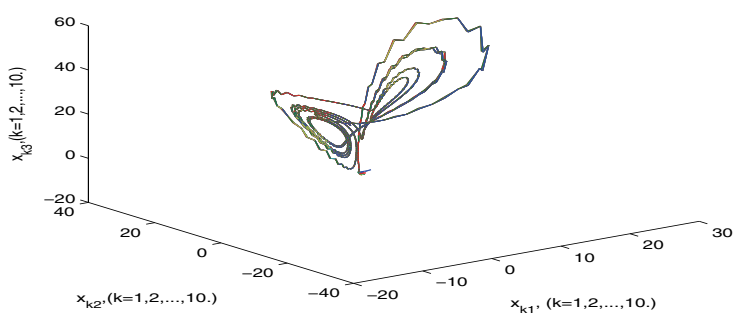

FIG. 6.8. The phase figure of the network under impulsive control.

In simulation, let $\tau=h_{i}=0.05, \Delta_{k}=0.03, k \in \mathbb{Z}$, and, without loss of generality, initial conditions $x_{i}(t)=0$ whenever $t<0$, and $x_{i}(0) \neq 0, i=1,2, \ldots, N$. The matrices $A_{i} \in N[\underline{A}, \bar{A}]$ are set as $A_{1}=A_{2}=A_{3}=\tilde{A}_{1}, A_{4}=A_{5}=A_{6}=\tilde{A}_{2}$, $A_{7}=A_{8}=\tilde{A}_{3}$, and $A_{9}=A_{10}=\tilde{A}_{4}$, where $\tilde{A}_{2}=A_{i_{0}}=A_{0}, \tilde{A}_{3}=\underline{A}, \tilde{A}_{4}=\bar{A}$, and

$$
\tilde{A}_{1}=\underline{A}+\operatorname{Rand}(3,3)=\left(\begin{array}{ccc}
0.4501 & 2.3860 & -0.0435 \\
-1.2689 & 0.1393 & -1.4815 \\
2.3068 & 2.4621 & 0.3214
\end{array}\right) .
$$

In Figures 6.4-6.6, one can see the exponential stability properties (at rate 0.0045 ) of errors between $s_{j}$ of the Lorenz system (6.3) and node states $x_{i j}, i=1,2, \ldots, 10$, $j=1,2,3$, of the CDN with $h_{1}=h_{2}=h_{3}=0.05$. In Figures 6.7 and 6.8 , one can see the whole consensus properties of Lorenz system (6.3) and the CDN (6.2) with coupling time-delay $h_{i}=0.05, i=1,2,3$.

7. Conclusions. In this paper, the global consensus problem has been studied between complex dynamical networks $(\mathrm{CDNs})$ and a known goal signal. The CDNs are more general than the usual models with respect to the uncertainties, nonidentical nodes, and coupling time-delays. We referred to the consensus scheme as an impulsive consensus scheme. By using the methods of Lyapunov functions and Lyapunov-Krasovskii functionals, as well as linear matrix inequalities (LMIs), criteria for achieving global exponential impulsive consensus (GEIC) have been derived. The impulsive consensus controllers so designed are effective in handing the uncertainties of parameters, coupling time-delays, and, more important, in synchronizing a known goal signal and a CDN with nonidentical nodes. The consensus rates of GEIC were also estimated. Finally, two examples have been discussed to illustrate the theoretical results obtained in this paper. 
Acknowledgments. The authors would like to thank the Editor, Professor Henk Nijmeijer, and the anonymous referees for their helpful comments and suggestions.

\section{REFERENCES}

[1] L. M. Pecora And T. L. Carroll, Synchronization in chaotic systems, Phys. Rev. Lett., 64 (1990), pp. 821-824.

[2] T. L. Carroll and L. M. Pecora, Synchronizing chaotic circuits, IEEE Trans. Circuits Syst., 38 (1991), pp. 453-456.

[3] L. Kocarev and U. PARLitz, General approach for chaotic synchronization with application to communication, Phys. Rev. Lett., 74 (1995), pp. 5028-5031.

[4] C. W. Wu, T. YANG, AND L. O. ChuA, On adaptive synchronization and control of nonlinear dynamical systems, Internat. J. Bifur. Chaos Appl. Sci. Engrg., 6 (1996), pp. 455-471.

[5] G. Grassi and S. Mascolo, Nonlinear observer design to synchronize hyperchaotic systems via a scalar signal, IEEE Trans. Circuits Systems I Fund. Theory Appl., 44 (1997), pp. 10111014.

[6] L. Kocarev, G. M. Maggio, M. Ogorzalek, L. Pecora, and K. Yao, Introduction to the special issue, IEEE Trans. Circuits Syst. I Regul. Pap., 48 (2001), pp. 1385-1388.

[7] G. Chen And X. Dong, On feedback control of chaotic continuous-time systems, IEEE Trans. Circuits Systems, 40 (1993), pp. 591-601.

[8] M. E. Yalcin, J. A. K. Suykens, and J. Vandewalle, Master-slave synchronization of Lur'e systems with time-delay, Internat. J. Bifur. Chaos Appl. Sci. Engrg., 11 (2001), pp. 17071772 .

[9] T. YANG AND L. O. ChUA, Impulsive stabilization for control and synchronization of chaotic systems: Theory and application to secure communication, IEEE Trans. Circuits Systems I Fund. Theory Appl., 44 (1997), pp. 976-988.

[10] H. M. Rodrigues, L. F. C. Alberto, and N. G. Bretas, On the invariance principle: Generalizations and applications to synchronization, IEEE Trans. Circuits Systems I Fund. Theory Appl., 47 (2000), pp. 730-739.

[11] A. Khadra, X. LiU, AND X. Shen, Application of impulsive synchronization to communication security, IEEE Trans. Circuits Systems I Fund. Theory Appl., 50 (2003), pp. 341-351.

[12] L. M. PeCORA AND T. L. CARroll, Master stability functions for synchronized coupled systems, Phys. Rev. Lett., 80 (1998), pp. 2109-2112.

[13] L. M. Pecora, T. L. Carroll, G. Johnson, D. Mar, and K. S. Fink, Synchronization stability in coupled oscillator arrays: Solution for arbitrary configurations, Internat. J. Bifur. Chaos Appl. Sci. Engrg., 10 (2000), pp. 273-290.

[14] C. W. WU AND L. O. ChUA, Synchronization in an array of linearly coupled dynamical systems, IEEE Trans. Circuits Systems I Fund. Theory Appl., 42 (1995), pp. 430-447.

[15] C. W. WU, Perturbation of coupling matrices and its effect on the synchronizability in arrays of coupled chaotic systems, Phys. Lett. A, 319 (2003), pp. 495-503.

[16] X. WANG AND G. CHEN, Synchronization in small-world dynamical networks, Internat. J. Bifur. Chaos Appl. Sci. Engrg., 12 (2002), pp. 187-192.

[17] X. WAng AND G. Chen, Synchronization in scale-free dynamical networks: Robustness and Fragility, IEEE Trans. Circuits Systems I Fund. Theory Appl., 49 (2002), pp. 54-62.

[18] J. Jost And M. P. Joy, Spectral properties and synchronization in coupled map lattices, Phys. Rev. E, 65 (2002), paper 016201.

[19] P. M. Gade AND C. K. Hu, Synchronous chaos in coupled map lattices with small-world interactions, Phys. Rev. E, 62 (2000), pp. 6409-6413.

[20] Z. Li And G. Chen, Robust adaptive synchronization of uncertain dynamical networks, Phys. Lett. A, 324 (2004), pp. 166-178.

[21] G. Chen, J. Zhou, AND Z. Liu, Global synchronization of coupled delayed neural networks and applications to chaotic CNN models, Internat. J. Bifur. Chaos Appl. Sci. Engrg., 14 (2004), pp. 2229-2240.

[22] C. Li And G. Chen, Synchronization in general complex dynamical networks with coupling delays, Phys. A, 343 (2004), pp. 263-278.

[23] C. LI, H. XU, X. LIAO, AND J. YU, Synchronization in small-world oscillator networks with coupling delays, Phys. A, 335 (2004), pp. 359-364.

[24] W. Lu And T. Chen, Synchronization analysis of linearly coupled networks of discrete time systems, Phys. D, 198 (2004), pp. 148-168.

[25] J. Lü AND G. Chen, A time-varying complex dynamical model and its controlled synchronization criteria, IEEE Trans. Automat. Control, 50 (2005), pp. 841-846.

Copyright (c) by SIAM. Unauthorized reproduction of this article is prohibited. 
[26] G. P. JiAng, W. K. S. TANG, And G. Chen, A state-observer-based approach for synchronization in complex dynamical networks, IEEE Trans. Circuits Syst. Regul. Pap., 53 (2006), pp. 2739-2745.

[27] J. Xiang and G. Chen, On the V-stability of complex dynamical networks, Automatica, 43 (2007), pp. 1049-1057.

[28] V. Lakshmikantham, D. D. Bainov, and P. S. Simeonov, Theory of Impulse Differential Equations, World Scientific, Singapore, 1989.

[29] D. D. Bainov And P. S. Simeonov, Systems with Impulsive Effects: Stability Theory and Applications, Halsted Press, New York, 1989.

[30] H. Ye, A. N. Michel, AND L. Hou, Stability analysis of systems with impulsive effects, IEEE Trans. Automat. Control, 43 (1998), pp. 1719-1723.

[31] T. YANG, Impulsive Control Theory, Springer-Verlag, Berlin, 2001.

[32] Z. G. Li, Y. C. Soh, And C. Y. Wen, Switched and Impulsive Systems: Analysis, Design and Application, Springer-Verlag, Berlin, 2005.

[33] Z. G. LI, C. Y. Wen, AND Y. C. SoH, Analysis and design of impulsive control systems, IEEE Trans. Automat. Control, 46 (2001), pp. 894-899.

[34] X. Z. Liu And J. H. Shen, Stability theory of hybrid dynamical systems with time delays, IEEE Trans. Automat. Control, 51 (2006), pp. 620-625.

[35] B. LiU, X. Z. LiU, G. Chen, And H. WANG, Robust impulsive synchronization of uncertain dynamical networks, IEEE Trans. Circuits Syst. I Regul. Pap., 52 (2005), pp. 1431-1441.

[36] B. LiU, X. Z. LIU, AND X. X. LiaO, Stability and robust stability of quasi-linear impulsive hybrid dynamical systems, J. Math. Anal. Appl., 283 (2003), pp. 416-430.

[37] B. Liu, X. Liu, AND K. L. TeO, Razumikhin-type theorems on exponential stability of impulsive delay systems, IMA J. Appl. Math., 71 (2006), pp. 47-61.

[38] B. Liu and H. J. Marquez, Razumikhin-type stability theorems for discrete delay systems, Automatica, 43 (2007), pp. 1219-1225.

[39] B. LiU, Stability of solutions for stochastic impulsive systems, IEEE Trans. Automat. Control, 53 (2008), pp. 2128-2133.

[40] B. LiU AND D. J. HilL, Impulsive consensus control for complex dynamical networks with nonidentical nodes and coupling time-delays, in Proceedings of the 47th IEEE Conference on Decision Control, Cancun, Mexico, 2008, pp. 2258-2263.

[41] J. K. Hale and S. M. Verduyn Lunel, Introduction to Functional Differential Equations, Springer, New York, 1993.

[42] D. J. Hill And J. ZhaO, Global synchronization of complex dynamical networks with nonidentical nodes, in Proceedings of the 47th IEEE Conference on Decision and Control, Cancun, Mexico, 2008, pp. 817-822.

[43] Y. M. Moon, P. Park, W. H. Kwon, And Y. S. Lee, Delay-dependent robust stabilization of uncertain state-delayed systems, Int. J. Control, 74 (2001), pp. 1447-1455.

[44] G. Chen, Controlling Chaos and Bifurcations in Engineering Systems, CRC Press, Boca Raton, FL, 2000.

[45] S. Boyd, L. El Ghaoui, E. Feron, and V. Balakrishnan, Linear Matrix Inequality in Systems and Control Theory, Stud. Appl. Math. 15, SIAM, Philadelphia, 1994.

[46] Z. H. Guan, D. J. Hill, And X. Shen, On hybrid impulsive and switching systems and applications to nonlinear control, IEEE Trans. Automat. Control, 50 (2005), pp. 1058-1062.

[47] M. V. BAsin And M. A. Pinsky, Stability impulse control of faulted nonlinear systems, IEEE Trans. Automat. Control, 43 (1998), pp. 1604-1608.

[48] M. V. BASIN AND M. A. PINSKY, On impulse and continuous observation control design in Kalman filtering problem, Systems Control Lett., 36 (1999), pp. 213-219.

[49] T. Zhang AND G. Feng, Output tracking of piecewise-linear systems via error feedback regulator with application to synchronization of nonlinear Chua's circuit, IEEE Trans. Circuits Syst. I Regul. Pap., 54 (2007), pp. 1852-1863.

[50] J. J. Hopfield, Neural networks and physical systems with emergent collect computational abilities, Proc. Natl. Acad. Sci. USA, 79 (1982), pp. 2554-2558.

[51] J. J. Hopfield And D. W. TANK, Computing with neural circuits-A model, Science, 233 (1986), pp. $625-633$.

[52] S. ARIK, An improved global stability result for delayed cellular neural networks, IEEE Trans. Circuits Syst. I Regul. Pap., 49 (2002), pp. 1121-1124.

[53] W. H. Chen And W. X. Zheng, Global exponential stability of impulsive neural network with variable delay: An LMI approach, IEEE Trans. Circuits Syst. I Regul. Pap., 56 (2009), pp. $1248-1259$.

[54] B. Liu, D. J. Hill, AND T. LiU, Synchronization of discrete-time CDN via delayed impulsive control, in Proceedings of the 8th IFAC Symposium on Nonlinear Control Systems (NOLCOS), Bologna, Italy, September 1-3, 2010, pp. 143-148.

Copyright (c) by SIAM. Unauthorized reproduction of this article is prohibited. 